\title{
Dynamic credit models
}

\author{
Stewart Inglis, Alex Lipton, Ioana Savescu and Artur Sepp
}

We present a dynamic framework to model the default events of individual obligors and the correlation between these default events. For the first purpose, we present the concepts of the dynamic jump-to-default model. For the second purpose, we concentrate on factor models which describe default events within a basket of obligors. In contrast to previous studies of factor credit models, we do not restrict ourselves to tractable, but not necessarily financially motivated, affine dynamics of the common factor and individual default intensities. Instead, we model the defaults using the logit survival function which depends on an appropriately chosen common factor. In the static version of the model, the distribution of the common factor is discrete, while in the dynamic version of the model the evolution of the common factor is driven by a jump-diffusion stochastic process. To solve the calibration and pricing problem, we develop robust partial integro-differential equation (PIDE) based numerical solution methods for the forward and backward Kolmogoroff equations. We also show how to augment the pricing problem with the loss intensity rate, and apply it to price structured credit products within the dynamic model. Finally, we provide an example of calibrating both the static and dynamic models to iTraxx credit index data.

Keywords AND PHRASES: Jump-to-default, Dynamic credit correlation, Factor correlation model.

\section{INTRODUCTION}

Credit risk is the potential loss experienced by the lender following the default or failure to pay of the borrower. In light of the recent financial turmoil triggered by the failure of US sub-prime loans, it has become apparent that the economic role of the credit market, which can be viewed as a continuum of lenders and borrowers, is difficult to overestimate. The market participants, who include investment banks, hedge funds, and insurance companies, increasingly are developing and implementing sophisticated analytical tools to value and risk-manage their credit risks on a day-today basis. Here, we will provide a detailed overview of some of the quantitative methods used to model credit risk. As we will demonstrate, these methods are derived from many well-known physical and mathematical models and often can be formulated as certain PDE problems.

The most basic security directly affected by a possible default of the borrower is the corporate bond. When this

contract is initiated, the borrower (or issuer) receives the initial notional of the bond from the lender; during the life of the bond, he pays to the lender appropriate coupons which represent the cost of using the loan, and on termination he returns the bond notional to the lender. We emphasize that the lender receives coupons and the notional only if the borrower honors his obligation; in the opposite case, the lender loses outstanding coupon payments and part of the notional. The possibility of the borrower's default is reflected in the spread measured as the difference between the rate of return on this bond as required by the lender and the rate of return on US treasury bonds, which are considered to be default-free securities. The spread charged on the borrower's bonds increases with the perceived probability of the default of this borrower.

When defaults do occur, lenders can suffer a mismatch between their incomes and expenses, which in the case of pension funds and insurers for example are often planned years ahead. To neutralise these credit risks, in the 1990s a contract developed which today has become one of the most prominent financial derivative securities - the credit default swap (CDS). In essence, the CDS contract provides its buyer with protection against a default of the contract-referenced issuer. The protection seller pays an amount commensurate with the respective loss experienced by the bond holder when the reference issuer defaults. In return, the protection buyer pays to the seller a periodic coupon conditioned on the survival of the referenced bond.

In an efficient capital market, the investor who owns both the bond and CDS protection should theoretically receive the risk-free rate of return, since he is clearly hedged against the issuer's default and therefore should receive no premium. This is not exactly the case in practice because of liquidity, tax and other issues. CDS contracts are however more liquid and transparent than the underlying bonds; protection sellers acquire credit risk exposure and financial compensation by receiving coupons conditioned on the survival of the referenced bond, and paying the loss if the issuer defaults. Next we introduce the collateralized debt obligation (CDO).

Institutional investors generally hold large portfolios of corporate bonds, and they might hedge their risk exposure by entering multiple CDS contracts referencing specific issuers. For large bond portfolios containing hundreds of bonds, however, it becomes very costly to maintain positions in dozens of CDS contracts. In the late 1990s, CDOs referencing large pools of single-name CDSs developed in order to help manage the credit risk in such portfolios. 
The investor who buys protection against defaults in a CDO contract pays periodic coupons whose magnitude depends on the number of survived issuers, and in return receives compensation (the loss given default) upon the default of individual issuers in the CDO pool.

The remarkable success of CDOs has resulted in the development of highly standardized credit indices, in particular, the CDX and iTraxx indices which respectively reference 125 US and 125 European corporate issuers where the notional of each issuer is $0.8 \%$ of the total notional of the index contract. These indices represent a large diversified pool of issuers, and they have become the benchmark for pricing and hedging basket products.

A position in the index contract yields to the investor the average coupon spread and the average credit quality of the constituent credit names. To satisfy the risk-reward appetite of more aggressive risk-taking, or conversely more modest risk-aversive investors, the market has devised the index tranches. Broadly speaking, the index tranches slice the coupon and loss distribution into three parts: the equity tranche that pays the highest coupon on its notional which, in turn, absorbs the first losses in the basket until it is completely exhausted; the mezzanine tranche which pays a less attractive coupon but whose notional starts depleting only after the equity tranche is wiped out; and the senior tranche, which is the most secure since its notional is affected by the basket losses only after both the equity and mezzanine tranches have been fully wiped out, and whose coupon is accordingly the lowest.

\subsection{Credit default swap}

We now introduce quantitative aspects of credit risk modeling by considering the basic credit default swap. For brevity we assume that a CDS contract has a notional of one currency unit, and denote the premium leg of the CDS by $P(t, T)$ and its default leg by $R(t, T)$, where $t$ is the current evaluation time and $T$ is the annualized maturity time of this CDS. We denote the annualized payment schedule associated with this CDS by $\left\{T_{i}\right\}_{i=0, . ., N}$, with $T_{0}=0$ and $T_{N}=T$, where $N$ is the total number of coupon payments, and we introduce annualized time periods $\Delta_{i}=T_{i}-T_{i-1}$, $i=1, \ldots, N$. Typically, the payment schedule is based on quarterly observations and the standardized maturity times of a CDS contract at inception are 1, 2, 3, 5 and 10 years.

The protection buyer of the CDS contract pays the coupon $\mathcal{S} \Delta_{i}$, where $\mathcal{S}$ is the CDS contract spread, at coupon time $T_{i}, i=1, \ldots, N$, conditioned on no default of the underlying reference up to time $T_{i}$ in exchange for protection equivalent to the percentage loss given default, $\mathcal{L}$, paid at time $T_{i}$ following the default of the reference name between $\left(T_{i-1}, T_{i}\right]$. The protection seller of the CDS contract at time $T_{i}$ receives a coupon of $\mathcal{S} \Delta_{i}$ units conditioned on the survival of the reference name, and pays the protection amount $\mathcal{L}$ upon the default event.
Now we introduce a key concept in credit risk modelingthe random default time $\iota$ of the CDS reference name. Without going into technical details, we assume that $\iota$ has support in $(0, \infty]$ and represent the cash flows of the premium leg, $\tilde{P}(t, T)$, as:

$$
\tilde{P}(t, T)=\mathcal{S} \sum_{i=1}^{N} \Delta_{i} \mathbf{1}_{\left\{\iota>T_{i}\right\}} \delta\left(t-T_{i}\right),
$$

where $\mathbf{1}_{\{.\}}$is the indicator function and $\delta(x)$ is the Dirac delta function.

The cash payment of the default leg, $\tilde{R}(t, T)$, can be represented as:

$$
\tilde{R}(t, T)=\mathcal{L} \delta(t-\iota) .
$$

To compute the expected values of the premium and default legs, we introduce the important notion of the survival probability of the reference name, $Q(t, T)$ :

$$
Q(t, T)=\mathbf{P}^{\mathbb{Q}}[\iota>T \mid \iota>t],
$$

where the expectation is computed under the pricing measure $\mathbb{Q}$. We assume that $\iota$ is independent from the dynamics of the risk-less interest rate and that the default event can occur only at coupon payment times $\left\{T_{i}\right\}_{i=1, \ldots, N}$. As a result, the expected present value of the payer leg, $P(t, T)$, is computed by:

$$
P(t, T)=\mathcal{S} \sum_{i=1}^{N} \Delta_{i} \mathcal{D}\left(t, T_{i}\right) Q\left(t, T_{i}\right),
$$

while the expected present value of the protection leg, $R(t, T)$, can be presented by:

$$
R(t, T)=\mathcal{L} \sum_{i=1}^{N} \mathcal{D}\left(t, T_{i}\right)\left(Q\left(t, T_{i-1}\right)-Q\left(t, T_{i}\right)\right),
$$

where $\mathcal{D}(t, T)$ is the time $t$ discount factor for a risk-less cash flow at time $T$.

The fair coupon $\mathcal{S}(T)$ for the CDS with maturity $T$ is computed by equating the corresponding premium and protection legs:

$$
\mathcal{S}(T)=\frac{\mathcal{L} \sum_{i=1}^{N} \mathcal{D}\left(t, T_{i}\right)\left(Q\left(t, T_{i-1}\right)-Q\left(t, T_{i}\right)\right)}{\sum_{i=1}^{N} \Delta_{i} \mathcal{D}\left(t, T_{i}\right) Q\left(t, T_{i}\right)}
$$

CDSs are quoted in the market in terms of their fair coupons (spreads) $\mathcal{S}(T)$. The single name CDS market is very liquid, so that given the term structure of CDS spread quotes we can also extract the term structure of the market implied default probabilities $\left\{Q\left(t, T_{i}\right)\right\}$ using equation (6), along with a piece-wise constant jump-to-default intensity specification (see Eq. (29)), which we discuss in more detail later. 
We note that our implied default probabilities depend on the choice of loss-given-default variable $\mathcal{L}$. While in principal $\mathcal{L}$ is a random variable and its modeling is studied in a huge number of papers (see chapter 6 in Schönbucher (2003) for an overview and a list of references), CDS market participants in practice assume a constant and pre-determined $\mathcal{L}$ which in most cases is $\mathcal{L}=60 \%$ (although the actual value of $\mathcal{L}$ upon the name's default is determined by the postdefault market value of the deliverable debt obligations). As a result, the market implied default probabilities can be extracted from CDS quotes, and these probabilities (together with the correlation between default times of different issuers) form one of the building blocks of CDO modeling.

\subsection{CDO tranches}

We now describe the pay-off of a tranche of an index backed by a pool of CDSs, with attachment and detachment points $a$ and $d$, where $0 \leq a<d \leq 1$, which determine the seniority of the tranche - the higher is $a$, the more secure is the index tranche. Let $L(t)$ denote the accumulated percentage loss of the pool at valuation time $t, 0 \leq L(t) \leq 1$, calculated as the total loss incurred up to time $t$ divided by the pool notional. Assuming that $\mathcal{L}$ is a constant for each of the underlying names then for index baskets the total loss is given by $L(t)=\mathcal{L} \frac{D(t)}{D_{\max }}$, where $D(t)$ is the number of defaults occurred up to time $t$ out of $D_{\max }$ names.

Let $L^{a, d}(t)$ denote the loss of the tranche at time $t$ which is calculated as follows:

$$
L^{a, d}(t)=\frac{1}{d-a}(\min (L(t), d)-\min (L(t), a)) .
$$

As for to the CDS contract, we denote the annualized payment schedule associated with this tranche by $\left\{T_{i}\right\}_{i=0 . . N}$, with $T_{0}=0$ and $T_{N}=T$, where $N$ is the total number of observations (fixings) and $T$ is the tranche maturity time, and we introduce annualized time periods $\Delta_{i}=T_{i}-T_{i-1}$ $i=1, \ldots, N$. Typically, the payment schedule is based on quarterly observations, and the standardized maturities of a tranche at inception are $3,5,7$, and 10 years.

In addition to an up-front payment $\mathcal{U}^{a, d}(T)$ paid per one percent of tranche notional at the contract inception, the premium leg of the index tranche pays at times $T_{i}$ a fixed coupon rate $\mathcal{S}^{a, d}(T)$ proportional to the remaining notional of the tranche at time $T_{i}$, so that the expected present value of the cash flows of the premium leg, $P^{a, d}(t, T)$, at time $t$ under the pricing measure $\mathbb{Q}$ can be presented by:

$$
\begin{aligned}
P^{a, d}(t, T)= & \mathcal{U}^{a, d}(T) \\
& +\mathcal{S}^{a, d}(T) \sum_{i=1}^{N} \Delta_{i} \mathcal{D}\left(t, T_{i}\right) \mathbb{E}^{\mathbb{Q}}\left[1-L^{a, d}\left(T_{i}\right)\right],
\end{aligned}
$$

where we assume that $L(t)$ is independent from the dynamics of the risk-free interest rate.

The default leg of the tranche pays at times $T_{i}, i=$ $1, \ldots, N$, tranche losses experienced between $\left(T_{i-1}, T_{i}\right]$, so that the expected value of cash flows of the default leg, $D^{a, d}(t, T)$, can be represented as:

$$
D^{a, d}(t, T)=\sum_{i=1}^{N} \mathcal{D}\left(t, T_{i}\right) \mathbb{E}^{\mathbb{Q}}\left[L^{a, d}\left(T_{i}\right)-L^{a, d}\left(T_{i-1}\right)\right] .
$$

The fair spread $\mathcal{S}^{a, d}$ equates payment and default legs and is given by:

$$
\begin{aligned}
& \mathcal{S}^{a, d}(T) \\
& \quad=\frac{-\mathcal{U}^{a, d}(T)+\sum_{i=1}^{N} \mathcal{D}\left(t, T_{i}\right) \mathbb{E}^{\mathbb{Q}}\left[L^{a, d}\left(T_{i}\right)-L^{a, d}\left(T_{i-1}\right)\right]}{\sum_{i=1}^{N} \Delta_{i} \mathcal{D}\left(t, T_{i}\right) \mathbb{E}^{\mathbb{Q}}\left[1-L^{a, d}\left(T_{i}\right)\right]} .
\end{aligned}
$$

We also note that for the full index, with $a=0$ and $d=1$, the loss in the premium leg is divided by $\mathcal{L}$ since the index notional is amortized by the number of defaults. We note that the maturities of CDOs and the underlying CDSs are typically different, and for the full index this effect (along with the poorer liquidity of underlying CDSs) causes what is known as the index basis, in which the average spread of CDSs underlying the index diverges slightly from the market quoted spread on the index, as opposed to the fact that in theory both of them should be equal.

For CDX and iTraxx indices, the equity tranche with $a=0$ and $d=3 \%$ is quoted in terms of the upfront payment with a fixed $5 \%$ annual coupon, while other tranches and the index are quoted in terms of their fair spreads. We note that the market quotes themselves provide little direct insight; however, given the set of spread quotes we can extract the term structure of the expected tranche losses which, among other things, indicates the implied probability of the tranche depletion. The corresponding procedure, which uses a modest assumption of linear interpolation of the expected losses across maturities, was proposed by Vacca (2005) and further enhanced by Lipton (2006).

Our key modeling challenge in valuing CDOs is how to model the loss distribution of baskets with many underlying credits. We first consider the models to describe single name defaults, and we then study how to model the correlation among single name defaults.

\section{MODELING INDIVIDUAL DEFAULT EVENTS}

\subsection{Merton model}

The seminal model which quantifies the relationship between company equity and debt and describes the company default event was proposed by Merton (1974). To introduce this model, we denote by $V(t)$ the company asset value per share at time $t$, by $D(t)$ the company obligation per share, which is approximately equal to the outstanding notional of the issued bonds per issued shares, and by $S(t)$ the company 
equity per share. In Merton's model, the company defaults at the debt repayment (maturity) time $T$ if the company value is less than its obligation at time $T, V(T)<D(T)$, which means that the company cannot honor its obligation.

Merton assumed that $V(t)$ is driven by the diffusion process:

$$
d V(t) / V(t)=\mu d t+\sigma d W(t)
$$

while the firm's obligation $D(t)$ is a deterministic function of time. Here, $W(t)$ is the standard Brownian motion, $\mu$ is constant drift and $\sigma$ is constant volatility.

In the Merton model, the default event can only occur at debt repayment time $T$ if the firm's obligation exceeds the firm's value, so that the default time is defined as follows:

$$
\iota= \begin{cases}T, & V(T) \leq D(T) \\ \infty, & V(T)>D(T)\end{cases}
$$

Accordingly, the probability of the firm's survival at time $T$, denoted by $Q(t, T)$, is given as follows:

$$
Q(t, T)=\mathcal{N}\left(d_{-}(t, V ; T, D)\right),
$$

where

$$
d_{ \pm}(t, V ; T, D)=\frac{\ln (V / D)+\left(r \pm \frac{1}{2} \sigma^{2}\right)(T-t)}{\sigma \sqrt{T-t}},
$$

and $\mathcal{N}(x)$ is the cumulative t.p.d.f. (transition probability density function) of the standard normal variable. The riskfree interest rate is $r$.

The value of the firm's equity at time $T$ can be viewed as the excess value after the obligation is paid:

$S(T)=(V(T)-D(T)) \mathbf{1}_{\{V(T)>D(T)\}}=\max (V(T)-D(T), 0)$,

while the value of its debt $B(t)$ at time $T$ is the minimum of the firm's value and its promised obligations:

$$
B(T)=D(T) \mathbf{1}_{\{V(T) \geq D(T)\}}+V(T) \mathbf{1}_{\{V(T)<D(T)\}} .
$$

Under the diffusion (11), the present value of the company equity discounted with the rate $r$ is the value of the call option on $V, S(t)=C(t, V ; T, D(T))$ where

$$
C(t, V ; T, D(T))=V \mathcal{N}\left(d_{+}\right)-e^{-(T-t) r} D(T) \mathcal{N}\left(d_{-}\right),
$$

while the the present value of the company equity discounted with the rate $r$ is:

(16)

$$
\begin{aligned}
B(t, V ; T, D(T)) & =V \mathcal{N}\left(-d_{+}\right)+e^{-(T-t) r} D(T) \mathcal{N}\left(d_{-}\right) \\
& =V(t)-C(t, V ; T, D(T))
\end{aligned}
$$

so that

$$
V(t)=C(t, V ; T, D(T))+B(t, V ; T, D(T)),
$$

214 S. Inglis et al. which is in agreement with the Nobel-prize winning Modigliani-Miller (1958) theorem which postulates that in an efficient market (that is one with no taxes, bankruptcy costs, or insider trading), the company value does not depend on whether its operations are financed through equity or debt. Although it is hard to challenge the statement of the theorem, the assumptions on which it is based generally do not hold in real markets.

Because the possibility exists of replicating the market value of the company by trading in its equity and bonds, it follows under the pricing (or the so-called martingale) measure that the return expected from taking the position in the firm's assets should be equal to the risk-free rate of return $r$. Accordingly, under the pricing measure the drift of the process (11) is $\mu=r$.

It is also very important to note that the Merton model is an equilibrium model - the model-predicted equity price should not necessarily be equal to the observed market price, so that the model is not consistent with the observable quantities unless it is properly calibrated. The important inputs to the Merton model are the volatility of the firm's value process $\sigma$ and the initial value of the company assets $V(t)$. These quantities are not directly observable in the market and are therefore backed-out from traded options on the company equity.

Subsequent important developments of the Merton model include Black-Cox (1976) and Leland-Toft (1996) who account for the possibility that the default may happen prior to the debt maturity time $T$. However, these models provide no connection to the equity market, and in particular to traded equity options which can be used for hedging the risk of the company default. We recall that the put (call) option on the stock share allows the investor to sell (buy) at the option maturity one share at a pre-agreed strike price. It is clear that when the company defaults and its equity price drops (close) to zero, the put option pay-off is its strike price, so that the equity put with a very low strike can be viewed as a credit product and its available market price can provide useful information for pricing and hedging other credit derivatives.

The direct link to the company equity was introduced by the CreditGrades (also known as the equity-to-credit) model that was jointly developed by CreditMetrics, JP Morgan, Goldman Sachs, and Deutsche Bank, and it has subsequently been copywritten (see Stamicar et al. (2005), Finger et al. (2002), Finkelstein (2001)). This model directly postulates that:

$$
V(t)=S(t)+D(t)
$$

where $V(t)$ is driven by diffusion (11), $S(t)$ is the equity per share directly observed from the share market price and $D(t)$ is the proxy for the company debt per share, which is typically estimated as $D(t)=R B(t)$ with $R, 0 \leq R<1$, being the recovery level of the company debt; usually $R \sim$ 
$40 \%-50 \%$. Typically we assume that the equity debt grows at the risk-free rate $r: B(t)=B e^{r t}$.

We note that since the CreditGrades model assumes that the firm's debt $D(t)=R B(t)$ is a fraction of the outstanding company obligations (due to bankruptcy costs, taxes etc. which decrease the value of the outstanding obligations), in general equation (17) is not satisfied and the CreditGrades model violates the Modigliani-Miller (1958) theorem.

In the CreditGrades model, the company defaults when $V(t) \leq D(t)$ or, equivalently, when $S(t)=0$, and the default time $\iota$ on the time interval $\left(t_{0}, T\right]$ is defined as the first passage time:

$$
\begin{aligned}
\iota & =\inf \left\{\tau \in\left(t_{0}, T\right]: V(\tau) \leq D(\tau)\right\} \\
& =\inf \left\{\tau \in\left(t_{0}, T\right]: S(\tau) \leq 0\right\}
\end{aligned}
$$

with $\inf \{\phi\}=\infty$ where $\phi$ is the empty set.

Representation (18) along with diffusion (11) is equivalent to the so-called displaced diffusion process introduced by Rubinstein (1983) with the t.p.d.f. of the asset price process given by:

$$
G\left(t, S ; T, S^{\prime}\right)=\frac{\mathfrak{n}\left(d_{-}\left(t, S+D ; T, S^{\prime}+D e^{(T-t) r}\right)\right)}{S^{\prime}+D e^{(T-t) r}},
$$

where $\mathfrak{n}(x)$ is the t.p.d.f. of the standard normal variable.

In Figure 1, we illustrate the important relationship between the probability density of $S(T)$ and the equity leverage coefficient $L=D(0) / S(0)$. Here, we show the t.p.d.f.-s of $S^{\prime}(T)$ corresponding to four choices of the leverage coefficient $L$ with $S(0)=1, R=0.5, B(0)=\{0,1,2,4\}$, $V(0)=\{1,1.5,2,3\}, L=\{0,0.5,1,2\}, T=1, r=0$ and $\sigma=0.3$.

We see that if the company does not have any debt its leverage is zero and there is no possibility of default on its obligation, so that $S^{\prime}(T)$ is always positive. For moderate

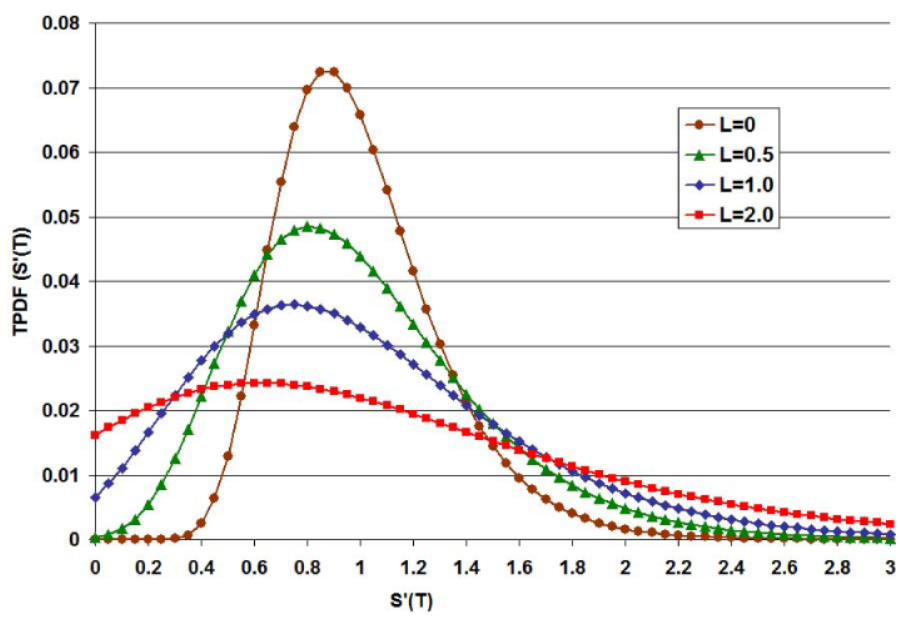

Figure 1. t.p.d.f. of the company equity price process under the CreditGrades model. values of the leverage the transition t.p.d.f. becomes more skewed with small probability of the default. Conversely, the greater the leverage, the more obligations the company has, so that the likelihood of defaulting on the debt and of $S^{\prime}(T)$ attaining zero increases. We note that with an increased leverage, the transition t.p.d.f. of the equity price also has a bigger right tail so that if things go well, equity holders can benefit by using an increased leverage. However, since the pay-off of the bond holders is bounded by the total notional and a higher default probability decreases the value of their positions, after a certain point they become reluctant to provide the company with new debt. Determining the optimal leverage has long been one of the keystones in both the theory and practice of finance (resulting in, among other interesting theories, the Modigliani-Miller (1958) theorem).

Since the equity holder's liabilities are always bounded by zero (the equity holder cannot lose more than he has invested), the company equity per share $S(t)$ is given by:

$$
S(t)= \begin{cases}V(t)-D(t), & \iota>t \\ 0, & \iota \leq t .\end{cases}
$$

Using (21), by pricing call and put options on the equity shares we have to augment the pricing problem with the barrier at zero. Thus, the value of the call option, denoted by $U(t, S)$, on the company shares solves the following problem:

$$
\left\{\begin{array}{l}
U_{t}+\frac{1}{2} \sigma^{2}(S+D)^{2} U_{S S}+r S U_{S}-r U=0 \\
U(t, 0)=0, U(T, S)=\max \{S-K, 0\}
\end{array}\right.
$$

while for the put option the value at zero is the strike price $K$ and at maturity is $\max \{K-S, 0\}$.

Applying the method of images we obtain the following solution to (22):

$$
\begin{aligned}
U(t, S)= & C\left(t, S+D ; T, K+D e^{(T-t) r}\right) \\
& -\frac{S+D}{D} C\left(t, \frac{D^{2}}{S+D} ; T, K+D e^{(T-t) r}\right),
\end{aligned}
$$

while the value of the put option is obtained by the put-call parity: $U^{p u t}(t, S)=U(t, S)-S+K e^{-(T-t) r}$.

The undiscounted survival probability also solves PDE (22) with zero discount factor, zero boundary condition and the terminal condition equal to one. Applying the method of images again, we obtain the survival probability to time $T$ :

$$
Q(t, T)=\mathcal{N}\left(d_{-}(t, V ; T, D)\right)-\frac{V}{D} \mathcal{N}\left(-d_{+}(t, V ; T, D)\right) .
$$

The CreditGrades model requires two main inputs: the company asset volatility $\sigma$ (this should not be confused with the equity price volatility) and the default barrier $D$. For a given maturity time, we typically back-out these parameters 
from the market price of an option and the survival probability implied by the market quote of the CDS contract. Properly calibrated, this model provides useful insight into the relationship between the company equity and debt and can be used for pricing and hedging of structured products such as convertible bonds.

The CreditGrades model however implies a very small probability of default at the start of the contract and therefore fails to explain the observations of short-term (up to two years) debt. To provide reconciliation with the observable market implied default probabilities, Zhou (1997) augmented the company value process (11) with log-normally distributed jumps. It turns out that the jump-process is able to explain high short-term default probabilities provided that the negative jumps in the asset value process are sufficiently large. Needless to say, the problem is more involved because of the non-local terms; Zhou's solution was based on Monte-Carlo, which is slow and noisy for model calibration. Lipton (2002) considers the log-exponentially distributed jumps and obtains the solution for the Laplace transform of the default probability, so that the problem becomes analytic up to the inversion of Laplace transform. Within the framework of the CreditGrades model, Sepp (2006) obtains solution for the call and put option values when the company value process is augmented with log-exponentially distributed jumps.

\subsection{Jump-to-default model}

Since the Merton model and its relatives always work with the company value process, which is not directly observable, researchers and practitioners have tried to model the default event by augmenting the asset price process with the fatal jump-to-default. Using the log-normal dynamics under the pricing measure $\mathbb{Q}$ we model the evolution of the asset price $S(t)$ as follows:

$$
d S(t) / S(t-)=(r+\lambda(t-, S)) d t+\sigma d W(t)-d N(t)
$$

where $N(t)$ is a Poisson process with intensity $\lambda(t, S)$, and $S(t-)$ is the asset price prior to the jump-to-default time. Following the first jump in $N(t)$ the asset price immediately drops to zero and the company is declared bankrupt.

Function $\lambda(t, S)$ serves as a means of connecting the default probability to the asset price, and in particular capturing the effect that low levels of the asset price are associated with higher default probabilities, and vice versa. The most common choice is the power function $\lambda(t, S)=\alpha\left(\frac{S(0)}{S(t)}\right)^{\beta}$ with $\alpha>0, \beta \geq 0$, where $\alpha$ is the overall level of the default intensity, $\beta$ is the skew factor.

The default time is the time of the first jump in the Poisson process $N(t)$ :

$$
\iota=\inf \{\tau>t: N(\tau)=1\} .
$$

Accordingly, the probability of survival at time $T$ in this model represents the probability of experiencing no jump in the process $N(t)$ :

$$
Q(t, T)=\mathbb{E}[N(T)=0 \mid N(t)=0]=\mathbb{E}\left[e^{-\int_{t}^{T} \lambda\left(t^{\prime}, S\right) d t^{\prime}}\right] .
$$

The value function of the derivative on $S, U(t, S)$, solves the following PDE:

$$
\begin{aligned}
U_{t} & +\frac{1}{2} \sigma^{2} S^{2} U_{S S}+(r+\lambda(t, S)) S U_{S}-(r+\lambda(t, S)) U \\
& =-\lambda(t, S) \bar{U}
\end{aligned}
$$

where $\bar{U}(t, S)$ is the option recovery value. In particular, the survival probability solves the PDE (28) supplied with boundary condition $U(T, S)=1$ and $\bar{U}(t, S)=0$; for the call option we again take $U(T, S)=\max \{S-K, 0\}$ and $\bar{U}(t, S)=0$, and for the put option $U(T, S)=\max \{K-S, 0\}$ and $\bar{U}(t, S)=K$.

To calibrate the model, we have to estimate the equity volatility $\sigma$ and $\alpha$ (the power parameter $\beta$ is typically chosen in advance by examining the historical data). For efficiency, Lipton (2003) and Andersen-Buffum (2003) solve the forward PDE adjoint to (28) and expressed as a function of $K$ and $T$ so that the model is calibrated by bootstrapping from the initial maturity up to the last available maturity. Linetsky (2006) applies an eigenfunction expansion method to solve this problem semi-analytically assuming that the model parameters are constant in time.

By applying a judicious discretization of the forward Kolmogoroff equation, in Figure 2 we are able to illustrate the t.p.d.f. of the equity price process for $S(0)=1, T=1$, $\alpha=0.01, \sigma=0.3, r=0$ using the power function with different values of $\beta$ coefficient. The probability mass at the origin represents the jump-to-default probability which increases as a function of $\beta$. No jump-to-default (No JTD)

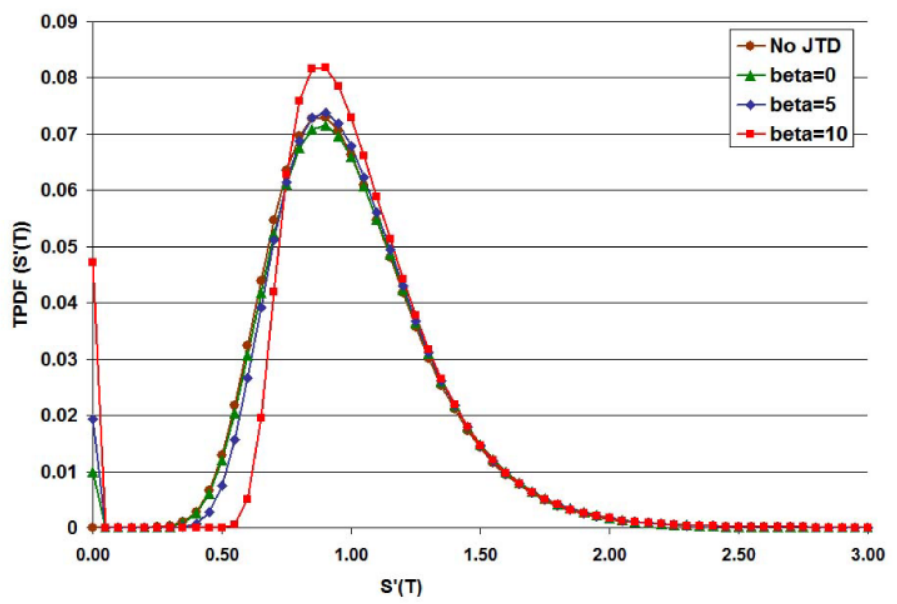

Figure 2. t.p.d.f. of asset price process with the jump-to-default. 
corresponds to the case $\lambda(t, S)=0$. We also see that a high value of beta reduces the left tail of the probability mass, implying that the default is more likely to occur when the stock price stays at low levels.

The jump-to-default model provides an important insight into the pricing problem. Its implied short-term default probability is sufficiently high and the model can be calibrated to reproduce the term-structure of implied default probabilities and market implied volatilities. It can also be extended to a multiple known or indeterminate number of jumps (Inglis-Tavella (2006)). Carr-Wu (2007) study a jump-to-default model with stochastic equity volatility which is capable of explaining the steep volatility skews observed in the market prices of equity options. Sepp (2007a) considers pricing and hedging options on the asset's realized variance under the jump-to-default model with stochastic volatility. It is evident that the jump-to-default model can be applied for pricing and hedging a variety of derivative contracts on different underlying assets, provided that the values of these contracts can be affected by defaults of issuers of the underlying assets.

\subsection{Reduced-form model}

The jump-to-default model works directly with the asset price and it requires non-linear calibration to produce the observable implied default probabilities and, as a result, reproduce the observable quotes for single name CDSs. In situations when we deal with large volumes of CDS contracts, the necessity of modeling the asset price directly becomes redundant and we can simplify the problem by assuming space-homogeneous deterministic default intensity rate $\lambda(t, S) \rightarrow \lambda(t)$, so that the survival probability given by equation (27) can be simplified considerably:

$$
Q(t, T)=e^{-\int_{t}^{T} \lambda\left(t^{\prime}\right) d t^{\prime}} .
$$

The typical approach in practice is to specify a piecewiseconstant term structure of the parameter $\lambda(t)$ and back it out from the observable market quotes of CDS contracts using equation (6). More generally, under the reduced-form model we specify the random evolution of the default intensity $\lambda(t)$ which in principle has no dependence on the actual evolution of the asset price (under the pricing measure).

\section{MODELING CORRELATED DEFAULTS}

Now we consider the main subject of our work - the modeling of correlated default events. Here we deal with several obligors, and our objective is to introduce the observed contagion effect, in which defaults tend to occur in clusters and which in turn implies high default correlations at certain (random) time periods.

\subsection{Multivariate models}

The first attempt to model the correlated default is the multivariate extension of the models we considered above. In the Merton model, we would introduce the correlated vector Brownian motion to model the vector of the asset value processes and infer the corresponding default probabilities. To illustrate the futility of this approach, we note that to model the default times of 125 issuers as required by our day-today operations, we would need to estimate 7750 correlation parameters and then solve a 125-dimensional problem! Even for a smaller basket this approach is fairly unreliable simply because the correlations implied from the time-series of equity prices, which fluctuate day by day, are poor proxies for the correlations of defaults, which are extremely rare events.

The straightforward extension of the reduced form model is also of little value because of the high dimensionality and lack of reliable data to estimate the correlations between the default events. The problem can however be simplified by sub-dividing the issuers in the given basket into relatively large groups, assuming that the default intensity for all the names in the sub-group is driven by the same intensity process, and then modeling the low-dimensional correlation structure.

We finish this subsection by noting that, in principal, the concept of the standard correlation applies for linear effects among the random variables, but the default events, besides being very rare, are empirically observed to occur in clusters. Thus, the usual correlation approach provides little, if any, insight for a problem at hand.

\subsection{Factor models and conditional independence}

Factor models provide a viable approach to model default correlation by assuming that a carefully chosen common factor affects the default intensities of all the issuers in some non-linear fashion. Conditional on the realization of this factor, the default events of single names in the basket are assumed to be independent, which enables fast computation of the conditional portfolio loss distribution. In general, we construct these models as follows.

1) We choose the distribution for the common factor $I$ which can be either discrete, continuous, or a mixture of both continuous and discrete parts, possibly timedependent. In general, we assume that $I \in[-\infty, \infty]$. Let $G(I)$ denote the probability density function of $I$.

2) Given the realization of the common factor $I(T)$ at time $T$, we denote the conditional survival probability at time $T$ given survival at $t, 0 \leq t \leq T<\infty$, conditioned on the realized market factor $I(T)$, of the credit name with index $k$ by the function:

$$
\mathfrak{Q}\left(T, I, \Theta_{k}\right)=\mathbb{E}^{\mathbb{Q}}\left[\mathbf{1}_{\left\{\iota_{k}>T\right\}} \mid \iota_{k}>t, I(T)\right],
$$


where $\iota_{k}$ is the default time of the $\mathrm{k}$-th credit name, $\Theta_{k}$ is the impact parameter (which is in general maturity dependent) or a set of parameters computed as explained below, and $\mathfrak{Q}\left(T, I, \Theta_{k}\right)$ is a non-linear function with the following property:

$$
\overline{\mathfrak{Q}}\left(t, T, \Theta_{k}\right):=\int_{-\infty}^{\infty} \mathfrak{Q}\left(T, I^{\prime}, \Theta_{k}\right) G\left(I^{\prime}\right) d I^{\prime}<\infty,
$$

where, in case the distribution of $I$ is discrete, the above integral is represented as the sum. In addition, we assume that $0 \leq \mathfrak{Q}\left(t, I, \Theta_{k}\right) \leq 1$ attaining its boundary values at the boundaries of the domain of $I$.

3) Then we introduce the unconditional expected survival probability of $k$-th name at time $t$ :

$$
\bar{Q}\left(t, T, \Theta_{k}\right)=\overline{\mathfrak{Q}}\left(t, T, \Theta_{k}\right),
$$

and compute the parameter $\Theta_{k}(T)$ at valuation time $t=0$ as the solution to the unconditional expected survival probability of $k$-th name given market implied survival probability $Q_{k}^{M}(0, T)$ :

$$
\bar{Q}\left(0, T, \Theta_{k}(T)\right)=Q_{k}^{M}(0, T),
$$

so that the expected theoretical survival probability is consistent with the market implied survival probability.

The conditional independence of the issuers allows the use of efficient algorithms to compute the credit portfolio loss distribution. The respective recursion algorithms have been used for many years in actuarial practice, and were introduced to the credit risk field by Andersen et al. (2003). In general, the distribution of losses in the credit portfolio consisting of $D_{\max }$ credit names at time $T$ is computed in the following way.

1) A relatively fine grid of the discrete state space, $\left\{I_{h}^{\prime}\right\}_{h=1 . . H}$, of the factor $I(T)$ at time $T$ along with corresponding probabilities, $\left\{P_{h}\right\}_{h=1 . . H}$, is constructed. We note that given the realization of $I(T)$, the default probabilities of individual names are independent among each other.

2) Given the market implied default probability and the discretisized distribution of $I(T)$, equation (32) is solved for each name $k, k=1 . . D_{\max }$, to obtain $\left\{\bar{Q}\left(0, T, \Theta_{k}(T)\right)\right\}_{k=1 . . D_{\max }}$. In general, this can be done applying Newton-Raphson solver.

3) For each state $I_{h}^{\prime}, h=1 . . H$, the portfolio loss distribution is computed using the independence of defaults and the default probability of individual name conditioned on given by $\mathfrak{Q}\left(T, I(T), \Theta_{k}\right)$ by means of the recursion procedure as follows. Let the conditional probability of experiencing $n$ defaults in $k$-name credit basket be denoted by $\Pi_{k}^{n}(I)$. This probability is computed as follows:

$$
\begin{aligned}
\Pi_{1}^{0}(I)= & \mathfrak{Q}\left(T, I, \Theta_{1}(T)\right), \Pi_{1}^{1}(I)=1-\mathfrak{Q}\left(T, I, \Theta_{1}(T)\right), \\
\Pi_{k}^{n}(I)= & \Pi_{k-1}^{n}(I) \mathfrak{Q}\left(T, I, \Theta_{k}(T)\right)+\Pi_{k-1}^{n-1}(I) \\
& \times\left(1-\mathfrak{Q}\left(T, I, \Theta_{k}(T)\right)\right), k=1 . . D_{\max }, n=1 . . k, .
\end{aligned}
$$

4) Finally, the unconditional probability of experiencing $n$ defaults in $k$-name credit basket is denoted by $\bar{\Pi}_{k}^{n}$ and is obtained by computing the average of the portfolio loss distributions obtained in step (3) weighted by the probability of the corresponding state as follows:

$$
\bar{\Pi}_{k}^{n}=\sum_{h=1}^{H} P_{h} \Pi_{k}^{n}\left(I_{h}\right) P_{h} \Delta I_{h}, n=1 . . k, k=1 . . D_{\max } .
$$

Given the distribution of portfolio losses we can then compute the expected tranche losses and, if needed, we can calibrate the model parameters by fitting the model implied losses to the market implied expected losses.

\subsubsection{Gaussian copula model}

We illustrate the above concepts by considering the industry standard Gaussian copula model introduced to the credit modeling field by Li (2000). In its essence, the Gaussian copula model is rooted in the Merton model for single name defaults, where we now assume that the $k$-th company defaults when

$$
\sqrt{1-\rho_{k}} X_{k}+\sqrt{\rho_{k}} I \leq \Theta_{k}
$$

where $X_{k}$ is a random variable specific to the $k$-th company, $\Theta_{k}$ is a deterministic default barrier of the $k$-th company, $I$ is the common factor specific to all the companies in the basket, and $\rho_{k} \in[0,1]$ is the correlation between the $k$ th company and the common market factor. Typically, we assume that $\rho_{k}$ is constant within the basket introducing the common correlation parameter $\rho: \rho_{k} \equiv \rho$.

In the standard Gaussian copula model both $X_{k}$ and $I$ are standard normal random variables, so that the probability of survival at time $T$ conditioned on the factor $I$ can be expressed using (36) as

$$
\mathfrak{Q}\left(T, I, \Theta_{k}\right)=\mathcal{N}\left(\frac{\sqrt{\rho} I-\Theta_{k}}{\sqrt{1-\rho}}\right),
$$

while the unconditional survival probability is $\overline{\mathfrak{Q}}\left(t, T, \Theta_{k}\right)=$ $1-\mathcal{N}\left(\Theta_{k}\right)$. Then we solve equation (33) to find $\Theta_{k}$ that matches the market implied survival probability $Q_{k}^{M}(0, T)$ for this maturity:

$$
\Theta_{k}(T)=\mathcal{N}^{-1}\left(1-Q_{k}^{M}(0, T)\right),
$$

which ensures that the model preserves the observed single name default probabilities.

When we use the Gaussian copula model to imply the correlation $\rho$ from the market quotes of the index tranches, we observe the remarkable base correlation skew effect, in which the correlation parameter increases with the tranche seniority level. Thus, under the Gaussian copula it is extremely difficult to handle all the tranches consistently even for a single maturity. There have been some attempts (for example, McGinty et al. (2004), Pain et al. (2005), Turc et al. (2006)) 
to extend the concept of the Gaussian copula correlation to the notion of base correlation by augmenting the Gaussian correlation with some scale and time dependence features to make the Gaussian copula fit the whole correlation surface. However, the Gaussian copula (like other copula-based models) can only be considered as a fitting method with no implied time-dynamics for the single name and index tranche spreads.

We refer to Andersen-Sidenius (2004), (2005), and HullWhite (2006) for a detailed study and references of copulabased factor models, and we forgo further discussion of them and concentrate instead on the static correlation model and its dynamic version.

\subsection{Static correlation model}

Now we discuss the static correlation model introduced by Lipton (2006) and Inglis-Lipton (2007). We note that in its principle the static correlation model is based on the reduced-form default model, where the default is triggered following jumps in the Poisson process, whereas the copula models are based on the Merton model, where default of the issuer is triggered when the firm value crossed the default barrier. It is interesting to note that while the reduced-form model is widely accepted for single name credits, the modeling of credit correlation is mostly performed within the copula extension of the Merton model.

The static correlation model uses the logit survival function:

$$
\mathfrak{Q}\left(T, I(T), \Theta_{k}(T)\right)=\frac{1}{1+e^{\Theta_{k}(T)+I(T)}}
$$

with impact parameter $\Theta_{k}(T)<0$.

We assume that the state of the realized default rate is discrete and has 4 states, $\mathbf{S}(I)=\left\{I_{0}, I_{1}, I_{2}, I_{3}\right\}$. The corresponding state probabilities are $\pi(I)=\left\{\pi_{0}, \pi_{1}, \pi_{2}, \pi_{3}\right\}$ with $\sum_{j=0}^{3} \pi_{j}=1$. To reduce the number of free parameters, we assume that $I_{0}=0$ and $I_{3}=\infty$. In this setup, $I_{0}, I_{1}$ and $I_{2}, I_{0}<I_{1}<I_{2}$, are interpreted as being three intermediate states of the economy, and $I_{3}=\infty$ can be interpreted as the "catastrophe" state in which every name in the basket defaults. The model has five free parameters to facilitate calibration to the five market quotes of the index tranches (a least-square optimization is required because the system of equations is highly non-linear).

Then given the market implied default probability $\left\{Q_{k}^{M}(0, T)\right\}$ equation (31) is solved for the implied value of $\left\{\Theta_{k}(T)\right\}$. We note that equation (31) now takes the form:

$$
\sum_{j=0}^{2} \frac{\pi_{j}}{1+e^{\Theta_{k}(T)+I_{j}}}=Q_{k}^{M}(0, T) .
$$

Since the left hand side of this equation is a concave function of $\Theta_{k}$ for $\Theta_{k}<0$, there exists a unique negative solution $\Theta_{k}^{*}$ to (40). This can be found by further representing (40) as a cubic equation and applying the Cardano formula to find its unique real-valued negative root.

Given the calibrated conditional survival function $\mathfrak{Q}\left(T, I(T), \Theta_{k}(T)\right)$, the portfolio loss distribution is computed by applying the recursion algorithm. Here, we have to apply the recursion only for three states since in the fourth state all names default and all tranches are depleted.

The static correlation model is capable of reproducing the implied loss distribution across all tranche seniority levels for a single maturity, and of providing a natural mapping for bespoke baskets.

\subsubsection{Large pool loss distribution}

To describe the asymptotic loss distribution in the credit portfolios with a large number of issuers where all insurers have the same survival probability $Q^{M}(T)$, we apply the Vasicek (1987) formula:

$$
\mathbb{P}\left[L \leq L^{\prime}\right]_{D_{\max } \rightarrow \infty}^{\longrightarrow} \mathbb{P}\left[1-Q(t, T) \leq L^{\prime}\right],
$$

where $L$ is the fractional loss, $L, L^{\prime} \in[0,1]$.

This asymptotic result is too approximate to be used for pricing, but it can be useful for risk-management purposes.

Applying this result for the Gaussian copula model we obtain:

$$
\mathbb{P}\left[L \leq L^{\prime}\right]=\mathcal{N}\left(\frac{\sqrt{1-\rho} \mathcal{N}^{-1}\left(L^{\prime}\right)-\mathcal{N}^{-1}\left(1-Q^{M}(0, T)\right)}{\sqrt{\rho}}\right) .
$$

For the static correlation model, we obtain:

$$
\mathbb{P}\left[L \leq L^{\prime}\right]=\sum_{k=0}^{3} \pi_{k} \mathbf{1}_{\left\{I_{k} \leq-\Theta+\ln \frac{L^{\prime}}{1-L^{\prime}}\right\}} .
$$

\section{DYNAMIC CORRELATION MODEL}

Inspired by the clarity and tractability of the static factor model, we now introduce the dynamics for the realized market intensity, which is denoted from now on by $I(t, T)$ to emphasize its dependence on time variable $t$. Instead of having one parameter to describe one market quote at a time, we introduce a set of time-dependent model parameters to describe a set of market quotes across all tranche structures and maturities.

\subsection{Market default intensity dynamics}

Following Lipton (2006), we introduce the following dynamics of the market default rate $\lambda(t)$ and the realized market default rate, $I(t)$, under the pricing measure $\mathbb{Q}$ :

$$
\begin{aligned}
d \lambda(t)= & \mu(t, \lambda(t)) d t+\sigma(t, \lambda(t)) d W(t)+J^{-} d N^{-}(t) \\
& +J^{+} d N^{+}(t)+J^{d} d N^{d}(t) \\
d I(t)= & F(t, \lambda(t)) d t, \lambda(0)=\lambda_{0}, I(0)=I_{0}
\end{aligned}
$$


where the mapping function $F(t, \lambda)$ is assumed to be positive.

Here, $W(t)$ is a standard Wiener process, and $\mu(t, \lambda)$ and $\sigma(t, \lambda)$ are drift and volatility functions of the market default rate.

$N^{+}(t)\left(N^{-}(t)\right)$ is a Poisson process with deterministic intensity $\gamma^{+}(t)\left(\gamma^{-}(t)\right)$ driving the arrival of positive (negative) jumps in the market factor. The magnitudes of these jumps, $J^{+}\left(J^{-}\right)$, have probability density function $\varpi^{+}(J)$ $\left(\varpi^{-}(J)\right)$ defined on the positive (negative) axis. Without loss of generality, we use the shifted-exponential jump-size distribution with t.p.d.f. given by:

$$
\varpi^{ \pm}(J)= \begin{cases}p^{ \pm} e^{\mp p^{ \pm}\left(J-q^{ \pm}\right)}, & \pm\left(J-q^{ \pm}\right) \geq 0, \\ 0, & \pm\left(J-q^{ \pm}\right)<0,\end{cases}
$$

where $q^{ \pm} \geq 0$ are shifts and $p^{ \pm} \geq 0$ are volatility parameters. In degenerate cases, if $q^{ \pm} \rightarrow 0$ then the jump density is exponential with mean $p^{ \pm}$, and if $p^{ \pm} \rightarrow 0$ then the jump density is the delta function peaked at $\pm q^{ \pm}$.

$N^{d}(t)$ is a Poisson process driving the jump-to-default with deterministic intensity $\alpha(t)$; upon the arrival of the first jump in $N^{d}(t)$ the market default rate along with the realized market default jump to infinity (informally, $J^{d}=\infty$ ) and all names in the basket default.

We note in our experience this specification of jump processes yields a sufficient degree of correlation in credit baskets, and it allows the calibration of strongly heterogeneous baskets. In general, the diffusion process of the market default rate captures the loss distribution of the full index and junior tranche, positive and negative jumps explain the distribution of losses in mezzanine tranches, and the jump-to-default determines the distribution of losses in senior tranches.

We also compare the static mixture model, which assumes static states of the realized default rate, with the dynamic model. In the dynamic setting, the states of the realized default rate are dynamic, and the distributions of $I_{0}$ and $I_{1}$ are determined by the diffusion part and negative jumps, the distribution of $I_{2}$ is determined by positive jumps and, finally, the probability of $I_{3}$ is determined by the jump-todefault. We emphasize that only this four-element formulation (diffusion plus three jumps) will provide close fits to market data, as in the static model with its four distinct states.

We denote by $\iota$ the random time of jump-to-default and we note that $\iota$ is an exponential random variable with hazard rate $\bar{\alpha}(t, T)=\int_{t}^{T} \alpha\left(t^{\prime}\right) d t^{\prime}$. We assume that all the information available at time $t$ about the market dynamics is contained in the augmented filtration $\mathcal{G}(t)=\mathcal{F}(t) \vee \sigma(\{\iota<\tau\}$, $\tau \leq t$ ), where $\mathcal{F}(t)$ contains information about intensity dynamics (44).

As we did for the static model, we assume that the conditional survival probability at time $T$ given survival at $t, 0 \leq t \leq T<\infty$, of a reference name with index $k$ is conditioned on, first, the realized market default rate $I(t, T)=\int_{t}^{T} F\left(t^{\prime}, \lambda\left(t^{\prime}\right)\right) d t^{\prime}$, and, second, on no jump-todefault up to time $T$ as defined by (30). Next we introduce the unconditional expected survival probability of $k$-th name at time $t$ :

$$
\begin{aligned}
\bar{Q}\left(t, T, \Theta_{k}(T)\right) & =\mathbb{E}^{\mathbb{Q}}\left[\mathbf{1}_{\{\iota>T\}} \mathfrak{Q}\left(T, I(t, T), \Theta_{k}(T)\right) \mid \mathcal{G}(t)\right] \\
& =\mathbf{1}_{\{\iota>t\}} e^{-\bar{\alpha}(t, T)} \overline{\mathfrak{Q}}\left(t, T, \Theta_{k}\right),
\end{aligned}
$$

where we employ the fact that $\iota$ is an exponential random variable independent of $\mathcal{F}(t)$.

The impact factor $\Theta_{k}(T)$ is now computed at time $t=0$ as a solution to the unconditional expected survival probability of the $k$-th name given the market implied default rate:

$$
\bar{Q}\left(0, T, \Theta_{k}(T)\right)=e^{\bar{\alpha}(0, T)} Q_{k}^{M}(0, T)
$$

so that the expected theoretical survival probability is consistent with the market implied survival probability.

For the conditional survival probability $\mathfrak{Q}\left(t, T, \Theta_{k}(T)\right)$ we also use the logit function (39). In other studies, a simpler exponential form is often used for the conditional survival probability. For example, the first dynamic factor correlation model introduced by Duffie-Garleanu (2001) is based on

$$
\mathfrak{Q}\left(T, I, \Theta_{k}\right)=e^{-\Theta_{k}(T) I(T)-I_{k}(T)}
$$

where the dynamic systematic factor $I$ and idiosyncratic factor $I_{k}$ are defined on the positive axes and $\Theta_{k}(T)$ is the correlation parameter. Duffie-Garleanu model is based on Monte-Carlo simulations, which has subsequently been improved by Mortensen (2006) who applied the AndersenSidenius-Basu (2003) recursion algorithm within the dynamic factor model with survival probability function given by (48). Similar models, but within one common factor and no idiosyncratic component, have been studied by Chapovsky et al. (2006), Sepp (2007b). These models assume that the common factor is driven by an affine process which along with the exponential form (48) allows the study of the pricing problem in "closed-form". Some computational aspects arising in the above models are considered by Eckner (2007).

Next we discuss the general pricing problem under the dynamic model, and after that we discuss the model calibration.

\subsection{General pricing problem under the dynamic model}

To solve the general pricing problem for exotic and path-dependent credit products depending on the realized losses as well as default intensities, we need to apply augmentation procedure (see Lipton (2001) for details) and augment the dynamics (44) with an auxiliary variable 
$\Lambda(t, \lambda, I, D, \Delta D)$ which describes the arrival of $\Delta D$ defaults, $\Delta D=1, \ldots, D_{\max }-D$, during the infinitesimal time interval $[t, t+\delta t]$ given the state of the dynamics at time $t$. Here, we let the random variable $D(t)$ stand for the total number of defaults in the portfolio up to time $t$; its space is $\mathbb{D}=\left\{0,1, \ldots, D_{\max }\right\}$. Also, we assume that the loss given the default of any credit name is a constant so that the portfolio loss distribution can be determined given the distribution of portfolio defaults.

In general, we need to consider the forward Kolmogoroff equation for the Green function $G^{\lambda I D}\left(t, T, \lambda, \lambda^{\prime}, I, I^{\prime}, D, D^{\prime}\right)$ describing the probability evolution of the state variables, and the backward Kolmogoroff equation for the value function, $U(t, T, \lambda, I, D)$, of a derivative security with payoff function $u_{1}(\lambda, I, D)$ at maturity time $T$ and reward function $u_{2}(t, \lambda, I, D)$ at time $t, 0<t<T$. These functions solve the following equations, respectively:

$$
\begin{aligned}
& G_{T}^{\lambda I D}+\left(\mu\left(T, \lambda^{\prime}\right) G^{\lambda I D}\right)_{\lambda^{\prime}}-\frac{1}{2}\left(\sigma^{2}\left(T, \lambda^{\prime}\right) G^{\lambda I D}\right)_{\lambda^{\prime} \lambda^{\prime}} \\
& \quad+\left(F\left(T, \lambda^{\prime}\right) G^{\lambda I D}\right)_{I^{\prime}}-\sum_{\Delta D=1}^{D_{\max }-D^{\prime}}\left(\Lambda\left(T, \lambda^{\prime}, I^{\prime}, D^{\prime}-\Delta D, \Delta D\right)\right. \\
& \left.\quad \times G^{\lambda I D}\left(D^{\prime}-\Delta D\right)-\Lambda\left(T, \lambda^{\prime}, I^{\prime}, D^{\prime}, \Delta D\right) G^{\lambda I D}\right) \\
& \quad-\gamma^{+}(T) \int_{0}^{\infty} G^{\lambda I D}\left(\lambda^{\prime}-J^{+}\right) \varpi^{+}\left(J^{+}\right) d J^{+} \\
& \quad-\gamma^{-}(T) \int_{-\infty}^{0} G^{\lambda I D}\left(\lambda^{\prime}-J^{-}\right) \varpi^{-}\left(J^{-}\right) d J^{-} \\
& \quad+\left(\gamma^{+}(T)+\gamma^{-}(T)+\alpha(T)\right) G^{\lambda I D}=0, \\
& G^{\lambda I D}\left(t, t, \lambda, \lambda^{\prime}, I, I^{\prime}, D, D^{\prime}\right)=\delta\left(\lambda^{\prime}-\lambda\right) \delta\left(I^{\prime}-I\right) \delta_{D^{\prime}, D}
\end{aligned}
$$

where $\delta$ is the Dirac delta function and $\delta_{D^{\prime}, D}$ is the Kronecker delta function with $\delta_{D^{\prime}, D}=1$ if $D^{\prime}=D$ and zero otherwise, and

$$
\begin{aligned}
& U_{t}+\mu(t, \lambda) U_{\lambda}+\frac{1}{2} \sigma^{2}(t, \lambda) U_{\lambda \lambda}+F(t, \lambda) U_{I} \\
& \quad+\sum_{\Delta D=1}^{D_{\max }-D} \Lambda(t, \lambda, I, D, \Delta D)(U(D+\Delta D)-U) \\
& \quad+\gamma^{+}(t) \int_{0}^{\infty} U\left(\lambda+J^{+}\right) \varpi^{+}\left(J^{+}\right) d J^{+} \\
& \quad+\gamma^{-}(t) \int_{-\infty}^{0} U\left(\lambda+J^{-}\right) \varpi^{-}\left(J^{-}\right) d J^{-} \\
& \quad-\left(r(t)+\gamma^{+}(t)+\alpha(t)\right) U=-u_{2}(t, \lambda, I, D), \\
& U(T, T, \lambda, I, D)=u_{1}(\lambda, I, D)
\end{aligned}
$$

where $r(t)$ is the deterministic interest rate.

We note that the transition rate function $\Lambda(t, \lambda, I, D$, $\Delta D)$ is specified implicitly by the state variables and model parameters. Next we briefly discuss the distinction between the bottom-up and top-down approaches to model the auxiliary function $\Lambda(t, \lambda, I, D, \Delta D)$, which measures the likelihood of experiencing $\Delta D$ defaults during a small time interval given that the current number of occurred defaults is $D$.

\subsection{The bottom-up approach}

Given $D_{\max }$ names in a basket, where each name has default intensity $\lambda_{k}(t)=-\left.\frac{\mathfrak{Q}\left(t+\delta t, I, \Theta_{k}\right)}{\delta t}\right|_{\delta t \rightarrow 0}$ conditioned on $\lambda(t), I(t)$, observing no catastrophic jump-to-default up to time $t$, we can model the default event of the $k$-th name by introducing a new binary random variable $L_{k}(t)$ with space $\{0,1\}$, where 0 stands for survival and 1 stands for default. Thus, $L_{k}(t)$ is driven by the following dynamics:

$$
d L_{k}(t)=d N_{k}(t), L_{k}(0)=0 \text { or } L_{k}(0)=1,
$$

where $N_{k}(t)$ is Poisson process with intensity (1 $\left.L_{k}(0)\right) \lambda_{k}(t)$

Accordingly, the number of occurred defaults $D(t)$ can be represented as:

$$
D(t)=\sum_{k=1}^{D_{\max }} L_{k}(t) .
$$

Conditioned on the realized market intensity rate and no jump-to-default, the distribution of $D(t)$ is given by nonhomogeneous Binomial distribution. Its dynamics can be written in the form:

$$
d D(t)=\sum_{k=1}^{D_{\max }} d L_{k}(t)=\sum_{k=1}^{D_{\max }} d N_{k}(t) \rightarrow d N_{D}(t),
$$

where $N_{D}(t)$ can be interpreted as a continuous time Markov chain with the transition matrix $\Lambda_{i j}(t, \lambda, I), i=$ $0, \ldots, D_{\max }, j=0, \ldots, D_{\max }$, with the following restrictions:

1) $\Lambda_{i j}=0, i>j, i=0, \ldots, D_{\max }, j=0, \ldots, D_{\max }$ (no recovery assumption);

2) $\Lambda_{i i}=-\sum_{j=i+1}^{D_{\max }} \Lambda_{i j}, i=0, \ldots, D_{\max }-1$, (balance equation);

3) $\Lambda_{D_{\max }, D_{\max }}=0$ (absorption in state $\left.D_{\max }\right)$.

Accordingly, the total number of admissible transition rates is $\frac{D_{\max }\left(D_{\max }+1\right)}{2}$. Following Chapovsky et al. (2006), we apply the additional restriction that the transition of defaults is possible only to the adjacent state (one default per infinitesimal time period), so that we have $\Lambda_{i j}=0$ for $j>i+1$. Thus, the transition matrix can be represented as bidiagonal matrix with $\Lambda_{i, i}=-\Lambda_{i}$ and $\Lambda_{i, i+1}=\Lambda_{i}, i=$ $0, \ldots, D_{\max }-1$, where $\Lambda_{i}$ is one-step transition rate from state $i$ to state $i+1$.

The one-step transition rates $\Lambda_{i}=\Lambda(t, \lambda, I, D), i=$ $0, \ldots, D_{\max }-1$, are assumed to be functions of time, the market default rate $\lambda$, realized rate $I$ and the number of occurred defaults $D$. 
Since conditional default probabilities are functions of $I$, the probability function of defaults conditioned on the instantaneous and realized intensity, $G^{D}\left(t, T, \lambda^{\prime}, I^{\prime}, D, D^{\prime}\right)$, satisfies the following Kolmogoroff forward equation:

(54)

$$
\begin{aligned}
& G_{T}^{D}+\left(F\left(T, \lambda^{\prime}\right) G^{D}\right)_{I^{\prime}}-\left(\Lambda\left(t, T, \lambda^{\prime}, I^{\prime}, D^{\prime}-1\right) G^{D}\left(D^{\prime}-1\right)\right. \\
& \left.\quad-\Lambda\left(t, T, \lambda^{\prime}, I^{\prime}, D^{\prime}\right) G^{D}\right)=0 \\
& G^{D}\left(t, t, \lambda^{\prime}, I^{\prime}, D, D^{\prime}\right)=\delta_{D^{\prime}, D} .
\end{aligned}
$$

Summing the left side in Eq. (54) from $D=0$ to $D=D^{\prime}$, and rearranging we obtain explicit solution for $\Lambda(t, T$, $\left.\lambda^{\prime}, I^{\prime}, D^{\prime}\right)$ :

$$
\begin{aligned}
\Lambda\left(t, T, \lambda^{\prime}, I^{\prime}, D^{\prime}\right)= & -\frac{1}{G^{D}\left(t, T, \lambda^{\prime}, I^{\prime}, D^{\prime}, D^{\prime}\right)} \\
& \times \sum_{D=0}^{D^{\prime}}\left(G_{T}^{D}+\left(F\left(T, \lambda^{\prime}\right) G^{D}\right)_{I^{\prime}}\right) .
\end{aligned}
$$

Once the full model has been calibrated and the conditional loss function $G^{D}\left(t, T, \lambda, I, D, D^{\prime}\right)$ has been computed on the discrete grid $\left\{T, \lambda^{\prime}, I^{\prime}, D^{\prime}\right\}$, we can apply (55) to retrieve $\Lambda\left(t, T, \lambda^{\prime}, I^{\prime}, D^{\prime}\right)$, which enables us to reproduce the distribution of losses at forward times. After that we use the backward equation (50) to price credit exotics.

The main advantage of this approach is that we do not lose information about single name credits, so that deltas and gammas are calculated by bumping spreads, calculating new implied default probabilities and then computing deltas and gammas by finite differences.

The main disadvantage of this method is that it requires extensive calculations. In situations when individual risks are not important, for example for options on tranches and structured basket products products we can apply a simplified version of the above approach by simplifying the function $\Lambda\left(t, T, \lambda^{\prime}, I^{\prime}, D^{\prime}\right)$. In general, the more restrictions we impose for $\Lambda\left(t, T, \lambda^{\prime}, I^{\prime}, D^{\prime}\right)$, the less likely we are to recover the initial implied loss surface. However, in situations when credit names in the basket can be considered as homogeneous we can apply a simplified top-down approach.

\subsection{The top-down approach}

In the top-down approach we make a crucial simplification by assuming that all names in the basket have the same (homogeneous) default probabilities. This contradicts the assumption of the full model, and to solve the calibration problem we do not consider computing the transition rate $\Lambda$ by means of Eq. (55) explicitly. Instead, we approach the problem by assuming, for brevity, a parametric function for $\Lambda$, and then perform the full calibration of default intensity dynamics (44) along with our chosen parametric function to the market data. Examples of non-parametric approaches

$$
d D(t)=\sum_{k=1}^{D_{\max }} d N_{k}(t) \rightarrow d N_{D}(t)
$$

where

to specifying the loss transition rate $\Lambda$ in the homogeneous dynamic model framework are considered by Arnsdorf et al. (2007) and Lopatin et al. (2007).

To illustrate this approach, we now assume that default variables $L_{k}(t), \quad k=1, \ldots, D_{\max }$, defined by (51) have homogeneous default intensity (51) $\lambda_{k}(t) \rightarrow$ $-\left.\frac{\mathfrak{Q}\left(t+\delta t, I, \Theta_{k}\right)}{\delta t}\right|_{\delta t \rightarrow 0}=F(t, \lambda)$ with common factor $\Theta$ conditioned on no jump-to-default up to time $t$. Given that conditioned on the realization of $F(t, \lambda)$, random variables $L_{k}(t)$ are independent, the dynamics of $D(t)$ specified by (53) can be represented by a Poisson process with intensity $\Lambda_{H}(t, \lambda, D)$ :

$$
\Lambda_{H}(t, \lambda, D)=\sum_{k=1}^{D_{\max }}\left(1-L_{k}(0)\right) \lambda_{k}(t)=\left(D_{\max }-D\right) F(t, \lambda),
$$

Accordingly, in homogeneous model given the explicit expression (57) for $\Lambda$, equation (54) can be written in the form:

$$
\begin{aligned}
& G_{I^{\prime}}^{D}-\left(D_{\max }-D^{\prime}-1\right) F(t, \lambda)\left(G^{D}\left(D^{\prime}-1\right)-G^{D}\right)=0, \\
& G^{D}\left(0, D, D^{\prime}\right)=\delta_{D^{\prime}, D},
\end{aligned}
$$

Given the realization of $I^{\prime}=\int_{t}^{T} F\left(t^{\prime}, \lambda\left(t^{\prime}\right)\right) d t^{\prime}$, the solution to Eq. (67) is defined by the negative binomial distribution (see, for example, Gnedenko (1998)), which in our setting assigns the probability of experiencing $D^{\prime}$ defaults and $D_{\max }-D^{\prime}$ survivals for $D_{\max }$ credit names along with the default at $D^{\prime}=D_{\max }$; it is given by:

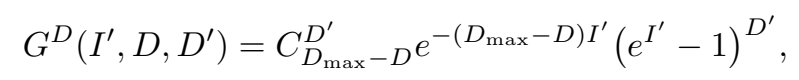

where $C_{N}^{k}=\frac{N !}{(N-k) ! k !}$.

\section{CALIBRATION OF THE DYNAMIC MODEL}

The framework we have discussed in the above section is fairly generic. As we have seen, it is often implemented by applying the tractable affine diffusions in the bottom-up or top-down approaches. However, we concentrate on the logit survival function (39) and develop robust numerical methods for the calibration and pricing problem.

\subsection{Calibration of the full model}

The implementation of the full dynamic factor model model consists of three steps. 
1) We calibrate the survival probabilities of single names by solving the following backward PDE:

$$
\begin{aligned}
& Q_{t}+\mu(t, \lambda) U_{\lambda}+\frac{1}{2} \sigma^{2}(t, \lambda) Q_{\lambda \lambda}+F(t, \lambda) Q_{I} \\
& +\gamma^{+}(t) \int_{0}^{\infty} Q\left(\lambda+J^{+}\right) \varpi^{+}\left(J^{+}\right) d J^{+} \\
& +\gamma^{-}(t) \int_{-\infty}^{0} Q\left(\lambda+J^{-}\right) \varpi^{-}\left(J^{-}\right) d J^{-} \\
& \quad-\left(\gamma^{+}(t)+\gamma^{-}(t)+\alpha(t)\right) Q=0, \\
& Q(T, T, \lambda, I)=\frac{1}{1+e^{I}} .
\end{aligned}
$$

Once the above equation is solved and the grid of values $Q(0, T, \lambda, I)$ is computed, we solve the calibration problem for single names by solving an algebraic equation to compute $\Theta_{k}(T)$ for each name:

$$
Q\left(0, T, \lambda, \Theta_{k}(T)\right)=Q_{k}^{M}(0, T) .
$$

2) We compute the density of the density of $I$ by solving the forward equation:

$$
\begin{aligned}
& G_{T}^{\lambda I}+\left(\mu\left(T, \lambda^{\prime}\right) G^{\lambda I}\right)_{\lambda^{\prime}}-\frac{1}{2}\left(\sigma^{2}\left(T, \lambda^{\prime}\right) G^{\lambda I}\right)_{\lambda^{\prime} \lambda^{\prime}} \\
&+\left(F(T, \lambda(T)) G^{\lambda I}\right)_{I^{\prime}} \\
&-\gamma^{+}(T) \int_{0}^{\infty} G^{\lambda I}\left(\lambda^{\prime}-J^{+}\right) \varpi^{+}\left(J^{+}\right) d J^{+} \\
&-\gamma^{-}(T) \int_{-\infty}^{0} G^{\lambda I}\left(\lambda^{\prime}-J^{-}\right) \varpi^{-}\left(J^{-}\right) d J^{-} \\
&+\left(\gamma^{+}(T)+\gamma^{-}(T)+\alpha(T)\right) G^{\lambda I}=0, \\
& G^{\lambda I}\left(t, t, \lambda, \lambda^{\prime}, I, I^{\prime}\right)=\delta\left(\lambda^{\prime}-\lambda\right) \delta\left(I^{\prime}-I\right),
\end{aligned}
$$

and computing the marginal density of $I$ by:

$$
G^{I}\left(t, T, \lambda, I, I^{\prime}\right)=\int_{0}^{\infty} G^{\lambda I}\left(t, T, \lambda, \lambda^{\prime}, I, I^{\prime}\right) d \lambda^{\prime} .
$$

3) Finally for each state, given the state-value of $I$ and the state probability $G^{I}$, we apply the recursion procedure to compute the portfolio losses for this state. The unconditional portfolio losses are obtained by weighting the stateconditional portfolio losses by the probability of the corresponding state.

\subsection{Calibration of the aggregated top-down model}

To solve the calibration problem under the aggregated model, we need to obtain solution to the Green function which solves the Kolmogoroff forward equation (49) with

$$
\Lambda\left(T, \lambda^{\prime}, I^{\prime}, D^{\prime}\right)=F\left(t, \lambda^{\prime}\right)\left(D_{\max }-D\right) .
$$

The one-factor Green function describing the evolution of defaults, $G\left(t, T, \lambda, I, D, D^{\prime}\right)$, is then given by:

$$
\begin{aligned}
& G\left(t, T, \lambda, I, D, D^{\prime}\right) \\
& \quad=\int_{0}^{\infty} \int_{0}^{\infty} G^{\lambda I D}\left(t, T, \lambda, \lambda^{\prime}, I, I^{\prime}, D, D^{\prime}\right) d \lambda^{\prime} d I^{\prime} .
\end{aligned}
$$

Then applying the idea of time-change we represent equation (65) in the form:

$$
\begin{aligned}
& G\left(t, T, \lambda, I, D, D^{\prime}\right) \\
& \quad=\int_{0}^{\infty} G^{D}\left(I^{\prime}, D, D^{\prime}\right) G^{I}\left(t, T, \lambda, I, I^{\prime}\right) d I^{\prime}
\end{aligned}
$$

with $G^{D}\left(0, D, D^{\prime}\right)=\delta_{D^{\prime}, D}$ and $G^{I}\left(t, t, \lambda, I, I^{\prime}\right)=\delta\left(I^{\prime}-I\right)$, and where we explicitly define $I^{\prime}$ as $I^{\prime}=\int_{t}^{T} F\left(t^{\prime}, \lambda\left(t^{\prime}\right)\right) d t^{\prime}$.

Plugging (66) into (49), we obtain the following equation for $G^{D}\left(I^{\prime}, D, D^{\prime}\right)$ :

(67)

$G_{I^{\prime}}^{D}-\left(\left(D_{\max }-D^{\prime}-1\right) G^{D}\left(D^{\prime}-1\right)-\left(D_{\max }-D^{\prime}\right) G^{D}\right)=0$,

$G^{D}\left(0, D, D^{\prime}\right)=\delta_{D^{\prime}, D}$,

with solution given by (59), and for $G^{I}\left(t, T, \lambda, I, I^{\prime}\right)$ with solution given by (63).

We compute the distribution of portfolio defaults (and thus portfolio losses) by means of discretisizing formula (66) as follows.

1) We discretisize the state space of $I^{\prime},\left\{I_{h}^{\prime}\right\}_{h=1 . . H}$, and compute the corresponding probabilities, $\left\{P_{h}\right\}_{h=1 . . H}$, by solving equation (62) along with (63).

2) For each state we compute the distribution of portfolio defaults by means of (59).

3) We compute the distribution of portfolio defaults as a weighted average of distributions obtained in 2) weighted by their respective state probabilities.

4) The probability of maximal number of defaults, $D_{\max }$, is augmented by $1-e^{-\bar{\alpha}(t, T)}$.

The model calibration is achieved by fitting the drift $\mu(t, \lambda)$ and volatility $\sigma(t, \lambda)$ of the aggregated intensity rate driven by dynamics (44) along with parameters of regular shocks and jump-to-default to a set of available tranches, and it is performed by applying the above procedure to match market quotes of these tranches. Once the aggregated intensity model is calibrated, we can calculate model prices of complex deals depending on the evolution of portfolio losses by solving the backward equation (50) or applying Monte-Carlo.

\section{NUMERICAL PIDE SOLUTION METHOD}

For the calibration and pricing methods to be numerically efficient, the numerical discretization of the forward and backward equations should be consistent. Here we outline the scheme developed by Lipton (2007). We first introduce the discrete time grid $\left\{t_{n}\right\}_{n=0 . . N}$ and spacial grids in 
$\lambda,\left\{\lambda_{k}\right\}_{k=0 . . K}$, and $I,\left\{I_{l}\right\}_{l=0 . . L}$, directions. For simplicity, we assume uniform grids with respective step sizes $\Delta t, \Delta \lambda$, and $\Delta I$.

We start with the backward equation and introduce the backward advection-diffusion operator $\mathfrak{D}_{\lambda}$ in $\lambda$ direction, the explicit jump-part operator $\mathcal{J}$, and explicit advection operator $\mathfrak{D}_{I}$ in $I$ direction. We then introduce the following discrete scheme for propagating the backward equation from the solution at time $t_{n+1}$ to the solution at time $t_{n}$ :

$$
Q^{n}=\left(\mathcal{I}+\mathfrak{D}_{\lambda}\right)^{-1}\left(\mathcal{I}-\mathfrak{D}_{\lambda}+\mathcal{J}\right)\left(\mathcal{I}+\mathfrak{D}_{I}\right) Q^{n+1} .
$$

We see that the discretization problem requires three steps which we consider below.

1) Discretization of advection operator in $I$-direction

This step is treated explicitly by using interpolation methods for hyperbolic equations. For the simple linear interpolation, the solution at node $I_{l}$ and $\lambda_{k}$ is computed as linear combination of solution at nodes $I_{l *}$ and $I_{l *-1}$ with $l *=\min \left(l^{\prime}>l: I_{l}+F\left(t_{n}, \lambda_{k}\right)>I_{l^{\prime}}\right)$.

2) Discretization of diffusion-advection operator in $\lambda$-direction

This step is treated by introducing the Crank-Nicolson discretization scheme that results in the tridiagonal matrix operator $\mathfrak{D}_{\lambda}$ :

$$
\mathfrak{D}_{\lambda}=\left(\begin{array}{ccccc}
B_{0} & C_{0} & 0 & 0 & \ldots \\
\ldots & \ldots & \ldots & \ldots & \ldots \\
\ldots & A_{k} & B_{k} & C_{k} & \ldots \\
\ldots & \ldots & \ldots & \ldots & \ldots \\
\ldots & 0 & 0 & A_{N} & B_{N}
\end{array}\right)
$$

where

(70)

$$
\begin{aligned}
A_{k} & =-\sigma\left(t_{n}, \lambda_{k}\right) \frac{\Delta t}{4(\Delta \lambda)^{2}}+\mu\left(t_{n}, \lambda_{k}\right) \frac{\Delta t}{4 \Delta \lambda}, \\
B_{k} & =\sigma\left(t_{n}, \lambda_{k}\right) \frac{\Delta t}{2(\Delta \lambda)^{2}}+\left(\gamma^{+}\left(t_{n}\right)+\gamma^{-}\left(t_{n}\right)+\alpha\left(t_{n}\right)\right) \frac{\Delta t}{2}, \\
C_{k} & =-\sigma\left(t_{n}, \lambda_{k}\right) \frac{\Delta t}{4(\Delta \lambda)^{2}}-\mu\left(t_{n}, \lambda_{k}\right) \frac{\Delta t}{4 \Delta \lambda} .
\end{aligned}
$$

We assume that for large values of $\lambda$, the Green function becomes linear in $\lambda$ so that $Q_{\lambda \lambda}=0$. As a result, the boundary condition yields:

$$
\begin{aligned}
& A_{0}=0, A_{N}=\mu\left(t_{n}, \lambda_{N}\right) \frac{\Delta t}{2 \Delta \lambda}, \\
& B_{0}=\left(\gamma^{+}\left(t_{n}\right)+\gamma^{-}\left(t_{n}\right)+\alpha\left(t_{n}\right)\right) \frac{\Delta t}{2}+\mu\left(t_{n}, \lambda_{0}\right) \frac{\Delta t}{2 \Delta \lambda}, \\
& B_{N}=\left(\gamma^{+}\left(t_{n}\right)+\gamma^{-}\left(t_{n}\right)+\alpha\left(t_{n}\right)\right) \frac{\Delta t}{2}-\mu\left(t_{n}, \lambda_{N}\right) \frac{\Delta t}{2 \Delta \lambda}, \\
& C_{0}=-\mu\left(t_{n}, \lambda_{0}\right) \frac{\Delta t}{2 \Delta \lambda}, \quad C_{N}=0 .
\end{aligned}
$$

3) Discretization of jump-part operator in $\lambda$ direction

Due to the special property of the shifted-exponential jumps, we can compute the jump part by a recursive algorithm (Lipton (2003), Toivanen (2006)). The key is to apply the sequence of operations of integration by parts and linear approximation of the derivative operator as follows:

$$
\begin{aligned}
I^{+} & (\lambda-\Delta \lambda)=p^{+} \int_{q^{+}}^{\infty} Q(\lambda-\Delta \lambda+j) e^{-p^{+}\left(j-q^{+}\right)} d j \\
= & p^{+} \int_{q^{+}-\Delta \lambda}^{\infty} Q(\lambda+j) e^{-p^{+}\left(j+\Delta \lambda-q^{+}\right)} d j \\
= & e^{-p^{+} \Delta \lambda} I^{+}(\lambda)+p^{+} e^{-p^{+} \Delta \lambda} \int_{-\Delta \lambda}^{0} Q\left(\lambda+q^{+}+j\right) e^{-p^{+} j} d j \\
= & e^{-p^{+} \Delta \lambda} I^{+}(\lambda)-e^{-p^{+} \Delta \lambda}\left[\left.Q\left(\lambda+q^{+}+j\right) e^{-p^{+} j}\right|_{-\Delta \lambda} ^{0}\right. \\
& \left.-\int_{-\Delta \lambda}^{0} Q_{j}^{\prime}\left(\lambda+j+q^{+}\right) e^{-p^{+} j} d j\right] \\
= & e^{-p^{+} \Delta \lambda} I^{+}(\lambda)-e^{-p^{+} \Delta \lambda} \frac{Q\left(\lambda+q^{+}\right)-Q\left(\lambda+q^{+}-\Delta \lambda\right)}{\Delta \lambda} \\
& \times\left.\frac{e^{-p^{+} j}}{p^{+}}\right|_{-\Delta \lambda} ^{0}-e^{-p^{+} \Delta \lambda} Q\left(\lambda+q^{+}\right)+Q\left(\lambda+q^{+}-\Delta \lambda\right) \\
= & e^{-p^{+} \Delta \lambda} I^{+}(\lambda)+Q\left(\lambda+q^{+}-\Delta \lambda\right)\left[1-\frac{1-e^{-p^{+} \Delta \lambda}}{p^{+} \Delta \lambda}\right] \\
& -Q\left(\lambda+q^{+}\right)\left[e^{-p^{+} \Delta \lambda}-\frac{1-e^{-p^{+} \Delta \lambda}}{p^{+} \Delta \lambda}\right] .
\end{aligned}
$$

A similar operation is performed for $I^{-}(\lambda+\Delta \lambda)$. As a result, the jump-part operator $\mathcal{J}$ is computed by descretisizing the (72) and the corresponding one for $I^{-}(\lambda+\Delta \lambda)$.

The discretization of evolution of the forward equation from solution at time $t_{n}$ to the solution at time $t_{n+1}$ consistent with the above scheme for the backward equation is given as follows:

$$
Q^{n+1}=\left(\mathcal{I}+\mathfrak{D}_{I}^{*}\right)\left(\mathcal{I}-\mathfrak{D}_{\lambda}^{*}+\mathcal{J}^{*}\right)\left(\mathcal{I}+\mathfrak{D}_{\lambda}^{*}\right)^{-1} Q^{n},
$$

where ${ }^{*}$ stands for the adjoint operator.

\section{CALIBRATION TO ITRAXX CREDIT INDEX}

To illustrate the above models, we calibrate them to the on-the-run series (S8) of European iTraxx credit index and index tranches. This index has three liquid maturities of 5,7 , and 10 years. In principle, we have some freedom in how we calibrate both the static and dynamic models, in particular, whether to make the model parameters time-dependent and calibrate the model to all three maturities. For simplicity, we provide calibration results in which we have fitted the model 
Table 1. Market quotes and static model calibration results for iTraxx Series 8, on the 7th of November, 2007

\begin{tabular}{c|c||c|c||c|c||c|c}
\hline \multicolumn{2}{c||}{ Attachment } & \multicolumn{2}{c||}{$5 \mathrm{Y}$} & \multicolumn{2}{c||}{$7 \mathrm{Y}$} & \multicolumn{2}{c}{$10 \mathrm{Y}$} \\
\hline Low & High & Market & Model & Market & Model & Market & Model \\
\hline \hline $0 \%$ & $3 \%$ & $21.75 \%$ & $21.79 \%$ & $29 \%$ & $29.07 \%$ & $36.875 \%$ & $36.86 \%$ \\
$3 \%$ & $6 \%$ & 150.5 & 152.2 & 210.5 & 210.6 & 377 & 377.5 \\
$6 \%$ & $9 \%$ & 72.5 & 72.9 & 108 & 108 & 158 & 158.2 \\
$9 \%$ & $12 \%$ & 52.5 & 52.7 & 72 & 71.9 & 104.5 & 104.5 \\
$12 \%$ & $22 \%$ & 32.5 & 36.8 & 46 & 47 & 63.5 & 64.4 \\
\hline \multicolumn{2}{l||}{ Index } & 49 & 49.03 & 56 & 56.03 & 65 & 65.03 \\
\hline
\end{tabular}

Table 2. Static model parameters

\begin{tabular}{l||c|c|c}
\hline & $5 \mathrm{Y}$ & $7 \mathrm{Y}$ & $10 \mathrm{Y}$ \\
\hline \hline$y_{1}$ & 1.631 & 1.465 & 1.241 \\
$y_{2}$ & 2.896 & 2.565 & 2.529 \\
$\pi_{1}$ & $90.83 \%$ & $84.21 \%$ & $54.04 \%$ \\
$\pi_{2}$ & $6.42 \%$ & $10.53 \%$ & $34.82 \%$ \\
$\pi_{3}$ & $1.38 \%$ & $2.63 \%$ & $6.88 \%$ \\
$\pi_{4}$ & $1.37 \%$ & $2.62 \%$ & $4.26 \%$ \\
\hline
\end{tabular}

parameters separately to each index maturity. We have used a constant $\mathcal{L}$ parameter equal to 0.62 , which is the average of the $\mathcal{L}$ values corresponding to the iTraxx underlying CDSs.

In Table 1 we provide the average bid/ask spreads (Market) quotes for iTraxx Series 8 IG observable on the 7th of November, 2007, and the corresponding model spreads (Model) implied by the static model. The equity tranche with attachment $0 \%$ and detachment $3 \%$ is quoted in terms of the upfront premium $\mathcal{U}$ expressed as a percentage of the notional together with a fixed coupon (or spread) of 500 basis point (one basis point is $0.01 \%$ ). All other tranches are quoted in terms of the fair spread expressed in basis points.

We see that the static model fits the market within the bid-ask spreads (except for the 5 year senior tranche, which is slightly outside the range). In Table 2, we show the calibrated parameters of the static model. We observe that the parameters increase/decrease monotonically in time (which is a desirable property). We also note that although we have five market observations to calibrate the five model parameters, the high non-linearity of the problem means that we require a multi-dimensional optimisation to fit the parameters (the system may have no exact solution).

In Figures 3 and 4 we show the loss density and the cumulative loss distribution implied by the static model. We truncate the normalized loss density at $20 \%$. Since the cumulative distributions do not intersect, the static model precludes arbitrage in the maturity dimension.

In Table 3 we provide the average bid/ask spreads (Market) quotes compared to the model implied spreads (Model) of the full dynamic model. For the dynamics of the market default intensity we use the Ornstein-Uhlenbeck process with $\mu(t, \lambda)=\kappa(\nu-\lambda)$ and $\sigma(t, \lambda)=\sigma$ with constant jumps amplitudes $q^{ \pm}= \pm 1$; for the mapping we use

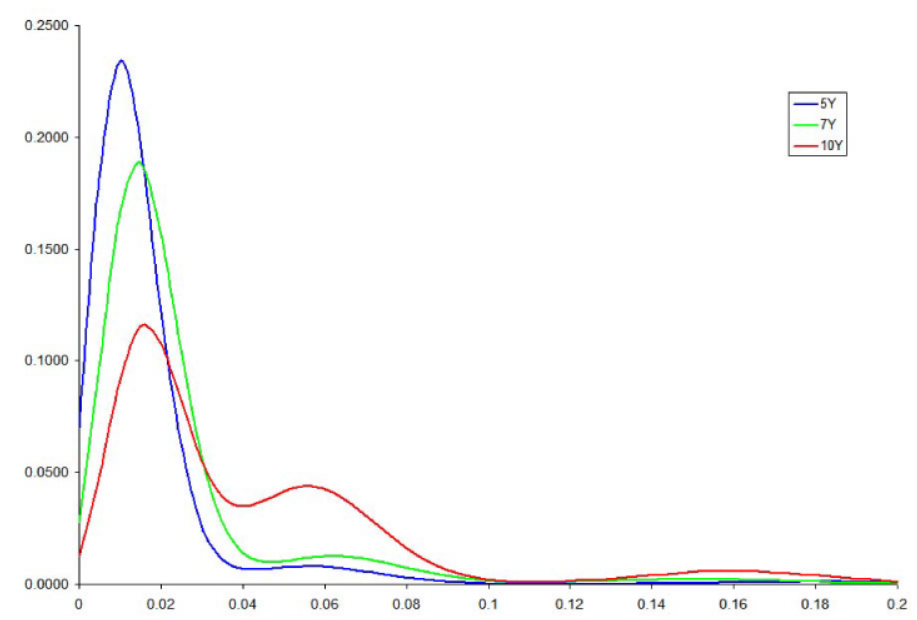

Figure 3. Loss density implied by the static model.

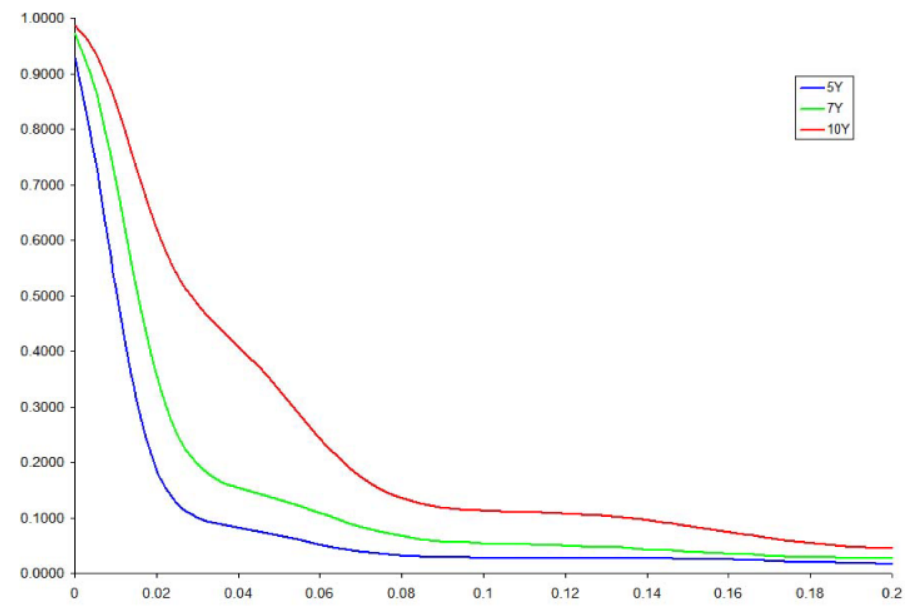

Figure 4. Cumulative loss distribution implied by the static model.

$F(t, \lambda)=0.25 e^{0.25 \lambda}$. The dynamic model also fits the market within the bid-ask spreads. We show its calibrated parameters in Table 4.

In Figures 5, 6 and 7 we show the density of the integrated market factor $I$, the loss density and the cumulative loss distribution implied by the dynamic model. 
Table 3. Market quotes and full dynamic model calibration results

\begin{tabular}{c|c||c|c||c|c||c|c}
\hline \multicolumn{2}{c||}{ Attachment } & \multicolumn{2}{c||}{$5 \mathrm{Y}$} & \multicolumn{2}{c||}{$7 \mathrm{Y}$} \\
\hline Low & High & Market & Model & Market & Model & Market & Model \\
\hline \hline $0 \%$ & $3 \%$ & $21.75 \%$ & $21.76 \%$ & $29 \%$ & $28.89 \%$ & $36.875 \%$ & $36.94 \%$ \\
$3 \%$ & $6 \%$ & 150.5 & 149.8 & 210.5 & 215.56 & 377 & 379.5 \\
$6 \%$ & $9 \%$ & 72.5 & 73.7 & 108 & 100.7 & 158 & 159.15 \\
$9 \%$ & $12 \%$ & 52.5 & 51.3 & 72 & 72.3 & 104.5 & 98.76 \\
$12 \%$ & $22 \%$ & 32.5 & 32.6 & 46 & 47.55 & 63.5 & 64.31 \\
\hline \multicolumn{2}{l}{ Index } & 49 & 46.65 & 56 & 53.64 & 65 & 63.36 \\
\hline
\end{tabular}

Table 4. Calibrated parameters for full dynamic model

\begin{tabular}{c||c|c|c}
\hline & $5 \mathrm{Y}$ & $7 \mathrm{Y}$ & $10 \mathrm{Y}$ \\
\hline \hline$\sigma$ & $46.8 \%$ & $30.00 \%$ & $48.24 \%$ \\
$\nu$ & 0.066 & -0.406 & -0.579 \\
$\kappa$ & 0.139 & 0.084 & 0.047 \\
$\gamma^{+}$ & $1.93 \%$ & $1.885 \%$ & $1.32 \%$ \\
$\gamma^{-}$ & $3.14 \%$ & $3.315 \%$ & $2.68 \%$ \\
$\alpha$ & $0.15 \%$ & $0.001 \%$ & $0.48 \%$ \\
\hline
\end{tabular}

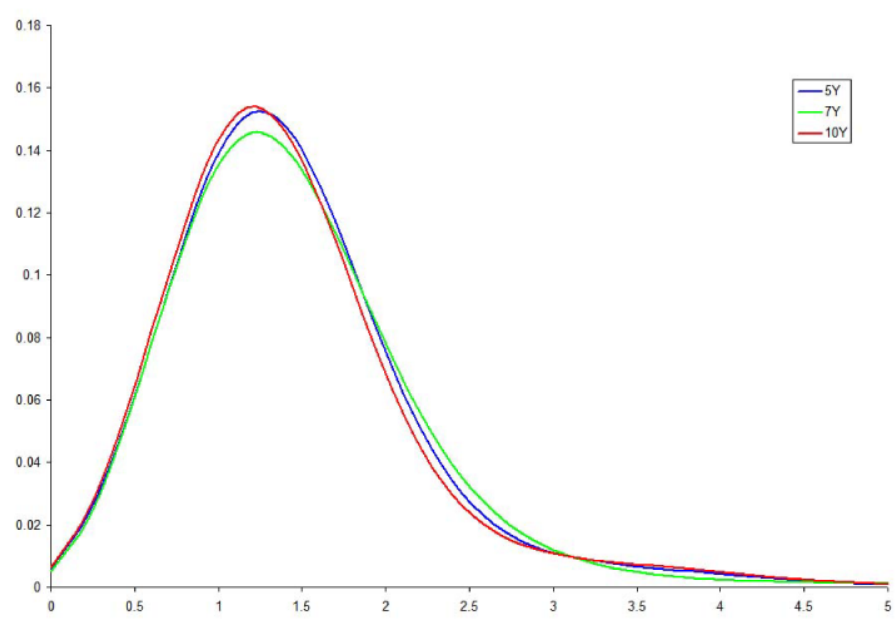

Figure 5. Density of the integrated factor I implied by the dynamic model.

\section{CONCLUSIONS}

We have presented a general framework to model default correlation using factor models. We have considered both static and dynamic models of the factor correlation model. Without restricting ourselves to tractable models, we have developed robust numerical solutions for the calibration and pricing problem. We have illustrated our models by calibrating them to iTraxx data.

Received 6 May 2008

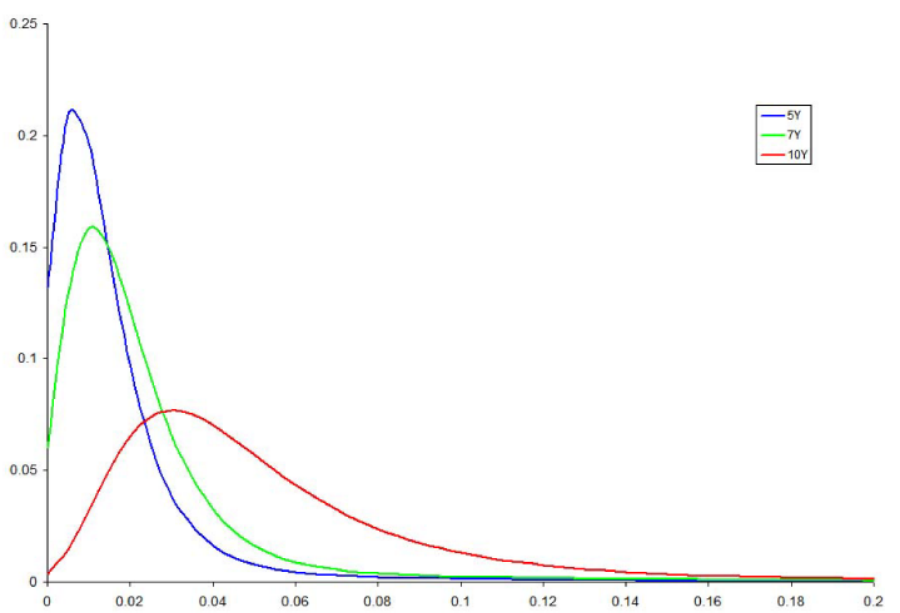

Figure 6. Loss density implied by the dynamic model.

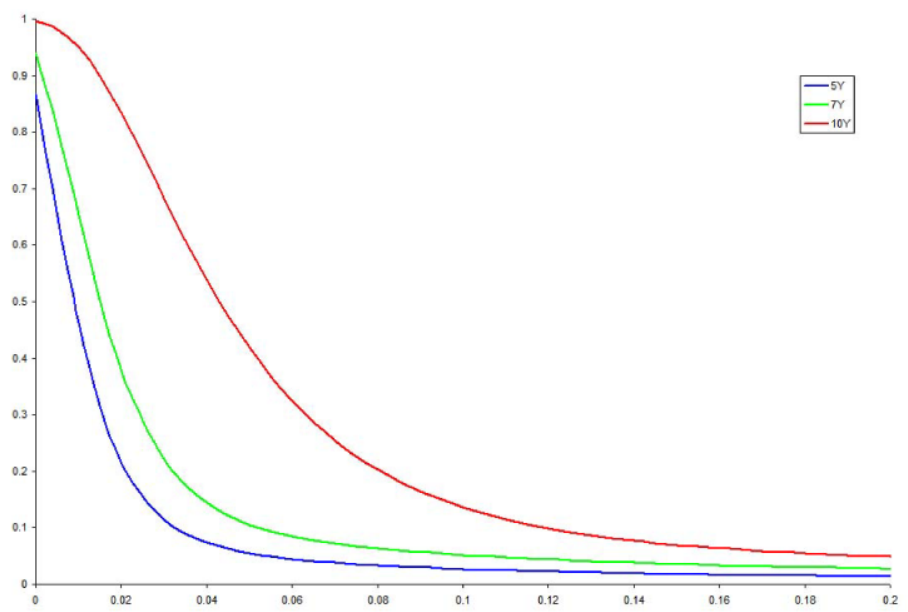

Figure 7. Cumulative loss distribution implied by the dynamic model.

\section{REFERENCES}

[1] Andersen L, Sidenius J 2005 CDO pricing with factor models: survey and comments (Journal of Credit Risk, 3(1), 71-88).

[2] Andersen L, Sidenius J 2004 Extensions of the Gaussian copula: random recovery and random factor loadings (Journal of Credit Risk, 1(1), 29-70). 
[3] Andersen L, Sidenius J, Basu S 2003 All your hedges in one basket (Risk, November, 67-72).

[4] Andersen L, Buffum D 2003 Calibration and Implementation of Convertible Bond Models (Banc of America Securities and Bank of America research paper).

[5] Arnsdorf M, Halperin I 2007 BSLP: Markovian Bivariate SpreadLoss Model for Portfolio Credit Derivatives (JP Morgan research paper).

[6] Black F, Cox J 1976 Valuing Corporate Securities: some effects of bond indenture provisions (Journal of Finance, 31, 351-367).

[7] Carr P, Wu L 2007 Theory and evidence on the dynamic interactions between sovereign credit default swaps and currency options (Journal of Banking and Finance, 31(8), 2383-2403).

[8] Chapovsky A, Rennie A, Tavares P 2006 Stochastic intensity modeling for structured credit exotics (International Journal of Theoretical and Applied Finance, 10(4), 633-652).

[9] Duffie D, Garleanu N 2001 Risk and valuation of collaterized debt obligations (Financial Analysts Journal, 57(1), 41-59).

[10] Eckner A 2007 Computational Techniques for Basic Affine Models of Portfolio Credit Risk (Stanford University research paper).

[11] Finger C, Finkelstein V, Pan G, Lardy J, Ta T, Tierney J 2002, CreditGrades technical document (RiskMetircs Group, available at www.riskmetrics.com).

[12] Finkelstein V 2001, Assessing Default Probabilities from Equity Markets (Presentation, Credit Risk Summit, NewYork).

[13] Gnedenko V 1998 Theory of Probability (CRC, 6th edition).

[14] Hull J, White A 2006 Valuing credit derivatives using an implied copula approach (Journal of Derivatives, 14(2), 8-28).

[15] Inglis S, Lipton A 2007 Factor models for credit correlation risk (Risk, 20(12)).

[16] Inglis S, Tavella D 2006 Pricing with Jump Signals in the PDE Framework (Wilmott, 24, 76-82).

[17] Leland H, Toft K 1996, Optimal capital structure, endogenous bankruptcy, and the term structure of credit spreads (Journal of Finance, 51, 987-1019).

[18] Lopatin A, Misirpashaev T 2007 Two-Dimensional Markovian Model for Dynamics of Aggregate Credit Loss (Numerix research paper).

[19] Li D 2000 On Default correlation: a copula approach (Journal of Fixed Income, (9), 43-54).

[20] Linetsky V 2006 Pricing equity derivatives subject to bankruptcy (Mathematical Finance, 16(2), 255-289). MR2212266

[21] Lipton A 2001 Mathematical Methods for Foreign Exchange: A Financial Engineer's Approach (World Scientific). MR1878627

[22] Lipton A 2002 Assets with jumps (Risk, September, 149-153).

[23] Lipton A 2003 Evaluating the Latest Structural and Hybrid Models for Credit Risk (Global derivatives conference in Barcelona).

[24] Lipton A 2006 Dynamic Credit Correlation Models: JumpDiffusion of the Market Factor and its Implications (Credit Risk Summit, Finance Concepts and Standard \& Poor's, 2006).

[25] Lipton A 2007 Pricing of Credit-Linked Notes and Related Products (Merrill Lynch research paper).

[26] McGinty L, Beinstein E, Ahluwalia R 2004 Introducing Base Correlation (JP Morgan research paper).

[27] Merton R 1974 On the pricing of corporate debt: the risk structure of interest rates (Journal of Finance, 29, 449-470).

[28] Modigliani F, Miller M 1958 The cost of capital, corporation finance and the theory of investment (American Economic Review, 48, 261-297).
[29] Mortensen A 2006 Semi-analytical valuation of basket credit derivatives in intensity-based model (Journal of Derivatives, 13, 8-26).

[30] Pain A, Renault O, Shelton D 2005 Base Correlation-the Term Structure Dimension (Citigroup research paper).

[31] Rubinstein M 1983 Displaced diffusion option pricing (Journal of Finance, 38(1), 213-217).

[32] Schönbucher P 2003 Credit Derivatives Pricing Models (Wiley Finance).

[33] Sepp A 2006 Extended CreditGrades model with stochastic volatility and jumps (Wilmott Magazine, September, 50-62).

[34] Sepp A 2007a Affine Models in Mathematical Finance: An Analytical Approach (PhD thesis, University of Tartu).

[35] Sepp A 2007b Dynamically Correlated Credits (Merrill Lynch research paper).

[36] Stamicar R, Finger C 2005 Incorporating Equity Derivatives into the CreditGrades Model (RiskMetrics Group, available at www.riskmetrics.com).

[37] Turc J, Benhamou D, Herzog B 2006 Pricing Bespoke CDOs-the Latest Developments (Societe Generale research paper).

[38] Toivanen J 2006 Numerical Valuation of European and American Options under Kou's Jump-Diffusion Model (Report B11/2006, Department of Mathematical Information Technology, University of Jyväskylä).

[39] Vacca L 2005 Unbiased risk-neutral loss distributions (Risk, November, 97-101).

[40] Vasicek O 1987 Probability of Loss on Loan Portfolio (Research paper, KMV corporation).

[41] Zhou C 1997 A Jump-Diffusion Approach to Modelling Credit Risk and Valuing Defaultable Securities (Federal Reserve Board, Washington).

\section{Stewart Inglis}

Merrill Lynch

4 World Financial Center

New York, NY 10080

E-mail address: Stewart_Inglis@ml.com

Alex Lipton

Merrill Lynch, Mlfc Main

2 King Edward Street

London, EC1A $1 \mathrm{HQ}$

E-mail address: Alex_Lipton@ml.com

loana Savescu

Merrill Lynch, Mlfc Main

2 King Edward Street

London, EC1A $1 \mathrm{HQ}$

E-mail address: Ioana_Savescu@ml.com

Artur Sepp

Merrill Lynch

4 World Financial Center

New York, NY 10080

E-mail address: Artur_Sepp@ml.com 